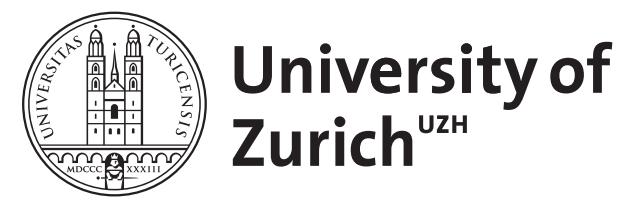

\title{
Stable mixture GARCH models
}

Broda, Simon A ; Haas, Markus ; Krause, Jochen ; Paolella, Marc S ; Steude, Sven C

\begin{abstract}
A new model class for univariate asset returns is proposed which involves the use of mixtures of stable Paretian distributions, and readily lends itself to use in a multivariate context for portfolio selection. The model nests numerous ones currently in use, and is shown to outperform all its special cases. In particular, an extensive out-of-sample risk forecasting exercise for seven major FX and equity indices confirms the superiority of the general model compared to its special cases and other competitors. Estimation issues related to problems associated with mixture models are discussed, and a new, general, method is proposed to successfully circumvent these. The model is straightforwardly extended to the multivariate setting by using an independent component analysis framework. The tractability of the relevant characteristic function then facilitates portfolio optimization using expected shortfall as the downside risk measure.
\end{abstract}

DOI: https://doi.org/10.1016/j.jeconom.2012.08.012

Posted at the Zurich Open Repository and Archive, University of Zurich

ZORA URL: https://doi.org/10.5167/uzh-55873

Journal Article

Accepted Version

Originally published at:

Broda, Simon A; Haas, Markus; Krause, Jochen; Paolella, Marc S; Steude, Sven C (2013). Stable mixture GARCH models. Journal of Econometrics, 172(2):292-306.

DOI: https://doi.org/10.1016/j.jeconom.2012.08.012 


\title{
Stable Mixture GARCH Models
}

\author{
Simon A. Broda ${ }^{\mathrm{a}}$, Markus Haas ${ }^{\mathrm{b}}$, Jochen Krause ${ }^{\mathrm{c}}$, Marc S. Paolella ${ }^{\mathrm{c}, \mathrm{d}, *}$, Sven C. Steude ${ }^{\mathrm{c}}$ \\ ${ }^{a}$ Department of Quantitative Economics, University of Amsterdam, Roetersstraat 11, 1018 WB \\ Amsterdam, Netherlands \\ ${ }^{b}$ Institute for Quantitative Business and Economics Research, University of Kiel, Heinrich-Hecht-Platz 9, \\ 24118 Kiel, Germany \\ ${ }^{c}$ Department of Banking and Finance, University of Zurich, Plattenstrasse 14, 8032 Zurich, Switzerland \\ ${ }^{d}$ Swiss Finance Institute
}

\begin{abstract}
A new model class for univariate asset returns is proposed which involves the use of mixtures of stable Paretian distributions, and readily lends itself to use in a multivariate context for portfolio selection. The model nests numerous ones currently in use, and is shown to outperform all its special cases. In particular, an extensive out-of-sample risk forecasting exercise for seven major FX and equity indices confirms the superiority of the general model compared to its special cases and other competitors. Estimation issues related to problems associated with mixture models are discussed, and a new, general, method is proposed to successfully circumvent these. The model is straightforwardly extended to the multivariate setting by using an independent component analysis framework. The tractability of the relevant characteristic function then facilitates portfolio optimization using expected shortfall as the downside risk measure.
\end{abstract}

JEL classification: C13; C16; C22; C32; G17

Keywords: Density Forecasting; Expected Shortfall; Fat Tails; ICA; GARCH;

Mixtures; Portfolio Selection; Stable Paretian Distribution; Value-at-Risk

\footnotetext{
*Corresponding author: marc.paolella@bf.uzh.ch

Email addresses: s.a.broda@uva.nl (Simon A. Broda), markus.haas@qber.uni-kiel.de (Markus Haas), jochen.krause@bf .uzh.ch (Jochen Krause), marc.paolella@bf.uzh.ch (Marc S. Paolella), sven.steude@bf.uzh.ch (Sven C. Steude)
} 


\section{Introduction}

Starting with the pioneering works of Mandelbrot (1963) and Fama (1965), a variety of studies have investigated the use of the stable Paretian distribution for modeling the unconditional distribution of asset returns. Given the very fat-tailed nature of weekly, daily, and higher-frequency financial returns data, it is not surprising that the stable distribution has been very successful in this regard. While there exist other fat-tailed, asymmetric distributions which also fit asset returns well, (see, e.g., Knight and Satchell, 2001; Paolella, 2007; and the references therein), none of these are closed under summation, a feature which is of great practical use in portfolio allocation; see e.g., Doganoglu et al. (2007) and Giacometti et al. (2007).

Despite the advantages of the stable distribution for modeling real phenomena, a popular, albeit often misguided, critique of the use of the stable Paretian distribution is the lack of existence of the second moment. (Recall that unless the tail index $\alpha \in(0,2]$ equals two, the tails are so heavy that absolute moments of order $\alpha$ and higher do not exist.) A number of studies have attempted to measure the tail index of the distribution of financial returns, as information about the tail index can be used to derive the probability of large price movements and, especially, market crashes (Jansen and de Vries, 1991). Additionally, knowing the maximum existing moment of the return process is of interest, as the lack of second moments will have consequences for risk and portfolio analysis. Nevertheless, it has been demonstrated by several authors that this endeavor is extremely difficult, so that no conclusive evidence of whether second moments of daily financial returns exist or not has been presented. For example, Kratz and Resnick (1996) discuss the inevitable and potentially "outrageous" bias inherent in tail thickness estimators such as the (in)famous Hill estimator (Hill, 1975).

The extreme bias problem for the Hill estimator with stable Paretian distributions has been studied in depth by McCulloch (1997), Mittnik et al. (1998) and Weron (2001). These and related studies have caused a shift from tail estimation to the adoption of a fat-tailed parametric assumption, such as the stable distribution. Indeed, this is embodied in the bold statement of Adler (1997), who states that "Many of the problems faced by the Hill and related estimators of the tail decay parameter $\alpha$ can be overcome if one is prepared to adopt a more parametric model and assume, for example, stable innovations". He goes on to say that "Overall, it seems that the time may have come to relegate Hill-like estimators to the Annals of Not-Terribly-Useful Ideas." With the above comments about tail estimation in mind (and Adler's positive stance towards use of the stable distribution), we know of no definitive way to test the existence or nonexistence of second moments. Based on the positive empirical findings below, we conclude that, for applications to density and risk forecasting, the question of existence of second moments is only of secondary interest. This stance is further supported by Malevergne et al. (2005) who state that "for most practical applications, the relevant question is not to determine what is the true asymptotic tail, but what is the best effective description of the tails in the domain of useful applications".

Another critique of the use of the stable Paretian distribution is the complexity of computing its density, as required for the likelihood - which for conditional (non-iid) models, is 
required for parameter estimation. With modern computing power, and the availability of several algorithms, this is no longer a hindrance. Nevertheless, there still appears to be no existing method which is both fast and delivers the high accuracy required for likelihood optimization. To this end, we propose yet another method for its computation. It capitalizes on the vectorized nature of modern computing languages and yields a method which is very fast, but also superior to existing algorithms in terms of accuracy. Its details, and references to other methods (and their flaws) can be found in the working version of this paper, Broda et al. (2011), hereafter WP.

The real problem with the use of the stable-Paretian, or any skewed, fat-tailed distribution for modeling the unconditional distribution of asset returns, is that they cannot capture the time-varying volatility so strongly evident in daily and higher-frequency returns data., ${ }^{1,2}$ Section 2 discusses the use of the stable distribution in conjunction with GARCH models to overcome this limitation.

Another popular and successful approach to the unconditional modeling of asset returns and $\mathrm{VaR}$ prediction involves the use of finite mixtures of normal distributions. Owing to its great flexibility, a normal mixture, even with just two components, is well-suited for capturing the usual stylized facts typical in a financial context. This model has been motivated and investigated by numerous authors, including Kon (1984), who suggests that returns may be influenced by a series of different information flows including a non-information distribution, a firm-specific information distribution, and a market-wide information distribution - hence, a mixture of three normal distributions. A different economic motivation for the presence of a mixture of distributions is provided by Vigfusson (1997), who builds on theoretical work which explains the stylized facts of financial time series by the interaction of heterogeneous groups of agents, with the groups processing market information differently; see,

\footnotetext{
${ }^{1}$ The term "volatility" is usually defined as the standard deviation of the log returns. In the models we shall be considering, the standard deviation is infinite, so this is strictly speaking a misnomer. In a slight abuse of terminology we shall continue to use the term and take it to refer to the (possibly time-varying) scale of the conditional return distribution.

${ }^{2}$ There is actually another problem with the stable Paretian (or mixtures thereof) which we do not address. The summability (or stability) property of the stable distribution and the definition of log returns implies that the tail index of the return distribution should remain the same at any frequency, i.e., intraday, daily, weekly, monthly, etc.. However, it is well-known that this is usually not the case, with, say, daily returns exhibiting a tail index considerably lower than two, but monthly data exhibiting nearly normal behavior. This occurs because, for such series, the returns are not iid stable Paretian, but rather have a distribution such that, via a central limit theorem, their sums approach normality. The iid aspect of this problem is addressed in Paolella (2001) by accounting for the non-constant scale term by application of a stable-GARCH filter and construction of a formal testing procedure, but even then, the null hypothesis of stability can be rejected for many (but not all) return series. This result is not in conflict with our stable mixture GARCH model because our goal is to (i) devise a model endowed with some plausible statistical and economic motivation, (ii) which yields relatively superior density and risk forecasts for daily (and possibly higher frequency) data, and (iii) can be used in a multivariate context via an ICA decomposition, but without concern for the stability (or lack thereof) aspect of returns. A possible way of incorporating all such features would be to use the tempered stable distribution, which also has a tractable characteristic function, mimics the shape of the stable distribution, but is such that, when iid copies are summed, the tail index increases; see Kim et al. (2008) and Kim et al. (2010).
} 
e.g., Samanidou et al. (2007) for an overview of such models. This is in line with recent research with experimental data by Kirchler and Huber (2007), who show that heterogeneous fundamental information can be a major source for the emergence of fat tails and volatility clustering. The fact that, for each component, a central limit theorem argument can be used to justify the use of the normal distribution is appealing, and lends some theoretical justification for the model and its economic interpretations. The same holds for the use of stable distributions for the mixture components, via the generalized central limit theorem.

The aforementioned problem regarding stable distributions also applies to the use of mixtures: in an unconditional setting, the mixture cannot capture the strong time-varying volatility of the returns. This is addressed by several authors who combine mixture models with GARCH structures. In this paper, we propose a model which generalizes the normality assumption in the normal mixture GARCH model to allow for stable distributions, investigate some of its theoretical and empirical properties, and extend its use (in a limited way adequate for portfolio optimization) to a multivariate framework. Moreover, as the estimation of all mixture models, and particularly mixture GARCH models, is numerically challenging due to the degeneracy problem, we introduce new estimators which elegantly resolve this. The degeneracy problem is illustrated graphically using real data, and the excellent performance of the new estimators is demonstrated via simulation.

The remainder of this paper is as follows. Section 2 introduces the new model. The new estimators are devised and outlined in Section 3. Section 4 presents an empirical exercise. Section 5 details how the model lends itself to portfolio allocation. Section 6 concludes.

\section{Stable Mixture GARCH}

Among conditional volatility models, the normal-GARCH has proven itself to be highly effective, though despite its success in capturing a high percentage of the volatility movement, countless applications have confirmed that the residuals, or filtered innovations when applied to weekly, daily, or higher frequency asset return data, still deviate considerably from normality. This has given rise to a large number of alternative models which replace the normal distribution in the GARCH model by a fat-tailed, asymmetric one. Given its theoretical properties, the stable Paretian distribution suggests itself, as first proposed by McCulloch (1985). See Mittnik et al. (2002) for further references and technical details, and Mittnik and Paolella (2003) for a demonstration of its effectiveness in value at risk (hereafter VaR) forecasting.

\subsection{Mixture GARCH}

A model which addresses the fat-tailed, asymmetric innovation issue mentioned above, but also gives rise to rich volatility dynamics not possible in the traditional battery of GARCH models, involves the use of mixtures. Building on the success of the mixed normal distribution for capturing the unconditional skewness and excess kurtosis of asset returns, and on some special cases already in the literature, Haas et al. (2004b) and Alexander and Lazar (2006) independently propose a general model structure which endows each mixed normal component with a GARCH structure. 
As in Haas et al. (2004b), we say that time series $\left\{\varepsilon_{t}\right\}$ is generated by a $k$-component mixed normal GARCH $(r, s)$ process, denoted MixNormal-GARCH, if the conditional distribution of $\varepsilon_{t}$ is a $k$-component mixed normal distribution with zero mean,

$$
\varepsilon_{t} \mid \mathcal{F}_{t-1} \sim \operatorname{MixNormal}\left(\boldsymbol{\omega}, \boldsymbol{\mu}, \boldsymbol{\sigma}_{t}\right)
$$

where $\boldsymbol{\omega}=\left(\omega_{1}, \ldots, \omega_{k}\right)^{\prime}, \boldsymbol{\mu}=\left(\mu_{1}, \ldots, \mu_{k}\right)^{\prime}$ and $\boldsymbol{\sigma}_{t}=\left(\sigma_{1, t}, \ldots, \sigma_{k, t}\right)^{\prime}$ are column vectors, the mixed normal probability density function (pdf) is given by

$$
f_{\varepsilon_{t} \mid \mathcal{F}_{t-1}}\left(x ; \boldsymbol{\omega}, \boldsymbol{\mu}, \boldsymbol{\sigma}_{t}\right)=\sum_{i=1}^{k} \omega_{i} \phi\left(x ; \mu_{i}, \sigma_{i, t}\right),
$$

$\mathcal{F}_{t}$ represents the information available at date $t, \phi$ is the normal pdf, $\omega_{i} \in(0,1)$ with $\sum_{i=1}^{k} \omega_{i}=1$ and, to ensure zero mean, $\mu_{k}=-\sum_{i=1}^{k-1}\left(\omega_{i} / \omega_{k}\right) \mu_{i}$. The component variances $\sigma_{i, t}^{2}$ follow the GARCH-like structure

$$
\boldsymbol{\sigma}_{t}^{(2)}=\boldsymbol{\gamma}_{0}+\sum_{i=1}^{r} \boldsymbol{\gamma}_{i} \varepsilon_{t-i}^{2}+\sum_{j=1}^{s} \boldsymbol{\Psi}_{j} \boldsymbol{\sigma}_{t-j}^{(2)}
$$

where $\gamma_{i}=\left(\gamma_{i, 1}, \gamma_{i, 2}, \ldots, \gamma_{i, k}\right)^{\prime}, i=0, \ldots, r$, are $k \times 1$ vectors, $\boldsymbol{\Psi}_{j}, j=1, \ldots, s$, are $k \times k$ matrices, $\boldsymbol{\sigma}_{t}^{(\delta)}$ is short for $\left(\sigma_{1, t}^{\delta}, \sigma_{2, t}^{\delta}, \ldots, \sigma_{k, t}^{\delta}\right)^{\prime}$, and $\delta \in \mathbb{R}_{>0}$. The parameters of the model need to be such that $\boldsymbol{\sigma}_{t}^{(\delta)}>0$, where, in case of non-scalars, > indicates element-wise inequality. As discussed in the above references, we take $r=s=1$ and restrict the $\boldsymbol{\Psi}_{j}$ to be diagonal, so in this case we will refer to the diagonal elements of $\boldsymbol{\Psi}_{1}$ as $\psi_{i}, i=1, \ldots, k$. Further details, and a survey of the model and the extensions which followed are given in Haas and Paolella (2011).

Another feature of mixture GARCH models is their ability to contain conditional as well as unconditional component models. As it turns out, the component of the mixture assigned to the most volatile observations can often be adequately modeled by a component with a relatively high, but constant, variance - it does not require a GARCH structure. We identify such models, where only $g, g \leq k$, components follow a $\operatorname{GARCH}(1,1)$ process, by appending the accessory $(k, g)$. Thus, the $k$-component MixNormal-GARCH model given in (1), with $r=s=1$ and $\boldsymbol{\Psi}_{1}=\operatorname{diag}\left(\psi_{1}, \ldots, \psi_{k}\right)$, is referred to as $\operatorname{MixNormal}(k, g)$.

Similar to replacing the normal assumption in a standard GARCH model, it suggests itself to challenge the MixNormal assumption with alternative distributions in addition to, or instead of, increasing the number of mixture components, to achieve better out of sample forecasting performance. As demonstrated in Kuester et al. (2006), one or more of the component densities of the MixNormal model can still exhibit tails which are fatter than the normal. In Kuester et al. (2006) and Rombouts and Bouaddi (2009) the generalized exponential distribution (in short, GED) is applied and shown to lead to improved in-sample fits and quality of VaR forecasts. For comparison with the MixStable-GARCH model, and using the aforementioned $(k, g)$ notation, we will refer to the MixGED-GARCH model as $\operatorname{Mix} \operatorname{GED}(k, g)$ in the following. 


\subsection{The MixStable Model}

Analogous to (1) and (2), we say that time series $\left\{\varepsilon_{t}\right\}$ follows a $k$-component mixed stable $\operatorname{GARCH}(r, s)$ process, denoted MixStable-GARCH, if the distribution of $\varepsilon_{t} \mid \mathcal{F}_{t-1}$ is a finite mixture of stable distributions. Its pdf is

$$
f_{\varepsilon_{t} \mid \mathcal{F}_{t-1}}\left(x ; \boldsymbol{\alpha}, \boldsymbol{\beta}, \boldsymbol{\omega}, \boldsymbol{\mu}, \boldsymbol{\sigma}_{t}\right)=\sum_{i=1}^{k} \omega_{i} f_{S}\left(x ; \alpha_{i}, \beta_{i}, \mu_{i}, \sigma_{i, t}\right),
$$

where $\boldsymbol{\alpha}=\left(\alpha_{1}, \ldots, \alpha_{k}\right)^{\prime}$ is the set of tail indices, $\boldsymbol{\beta}=\left(\beta_{1}, \ldots, \beta_{k}\right)^{\prime}$ is the set of asymmetry parameters corresponding to the $k$ stable distributional components, and, as before, $\boldsymbol{\omega}=$ $\left(\omega_{1}, \ldots, \omega_{k}\right)^{\prime}$ is the set of weights, $\boldsymbol{\mu}=\left(\mu_{1}, \ldots, \mu_{k}\right)^{\prime}$ is the set of component location terms, $\boldsymbol{\sigma}_{t}=\left(\sigma_{1, t}, \ldots, \sigma_{k, t}\right)^{\prime}$ is the set of strictly positive scale parameters, and $f_{S}(x ; \alpha, \beta, \mu, \sigma)$ is the location- $\mu$, scale- $\sigma$, stable Paretian pdf with tail index $\alpha$ and skewness parameter $\beta$, as in Samorodnitsky and Taqqu (1994). For the mixture GARCH, let $\boldsymbol{\delta}=\left(\delta_{1}, \ldots, \delta_{k}\right)^{\prime}$ be the set of power GARCH coefficients. Further, we assume that $1<\alpha_{i} \leq 2$, so that the mean exists, and, with $\alpha_{\min }=\min _{i} \alpha_{i}$, restrict $0<\delta_{i}<\alpha_{\min }, i=1, \ldots, k$. This is a natural extension of the power restriction in the stable-GARCH model as devised in Mittnik et al. (2002). If $\alpha_{\min }=2$ (so that $\alpha_{i}=2$ for all $i$ ), the $\delta_{i}$ need only be positive, $i=1, \ldots, k$. As with $\operatorname{MixNormal}(k, g)$, to ensure zero mean, $\mu_{k}=-\sum_{i=1}^{k-1}\left(\omega_{i} / \omega_{k}\right) \mu_{i}$ is imposed. The component scale terms, analogous to the variance term in the MixNormal model, evolve according to

$$
\boldsymbol{\sigma}_{t}^{(\boldsymbol{\delta})}=\boldsymbol{\gamma}_{0}+\sum_{i=1}^{r} \boldsymbol{\gamma}_{i}\left|\varepsilon_{t-i}\right|^{(\boldsymbol{\delta})}+\sum_{j=1}^{s} \boldsymbol{\Psi}_{j} \boldsymbol{\sigma}_{t-j}^{(\boldsymbol{\delta})},
$$

where $\boldsymbol{\sigma}_{t}^{(\boldsymbol{\delta})}$ is short for $\left(\sigma_{1, t}^{\delta_{1}}, \sigma_{2, t}^{\delta_{2}}, \ldots, \sigma_{k, t}^{\delta_{k}}\right)^{\prime}$. Motivated by our use of relatively (for GARCH applications) small sample sizes (see the comment in Footnote 9 and the discussion in Section 4.3 below), we impose $\alpha_{i}=\alpha_{j}$ and $\beta_{i}=\beta_{j}$ for all stable mixture components as well as $\delta_{i}=\delta_{j}$ for all mixture GARCH models in the following. As such, we drop the component index $i$ and just write $\alpha, \beta$ and $\delta$. Similar to $\operatorname{MixNormal}(k, g)$, MixStable $(k, g)$ denotes the $k$-component mixed stable $\operatorname{GARCH}(1,1)$ process with diagonal $\boldsymbol{\Psi}_{1}$ matrix, and only $g$ of the $k$ components having a GARCH structure. For $\beta=0$, the model nests the following:

1) The unconditional stable Paretian model, as proposed by Mandelbrot (1963) and Fama (1965), by taking $k=1$ and no GARCH structure.

2) The unconditional mixed normal model from Fama (1965), Kon (1984) and others, by taking $\alpha_{i}=$ in each of the $k$ components, and no GARCH structure.

3) The normal-GARCH model from Bollerslev (1986), by taking $k=1, \alpha=2$ and $\delta=2$.

4) The symmetric stable-GARCH model of Mittnik et al. (2002), by taking $k=1$.

5) The MixNormal $(k, g)$ model (1) and (2) of Haas et al. (2004b) and Alexander and Lazar (2006), by taking $\delta=2$ and $\alpha=2$. 
6) The "linear" two-component MixNormal-GARCH models of Vlaar and Palm (1993) and Bai et al. (2003).

The MixStable-GARCH model is very general; in particular, there are two sources of asymmetry in the model: $\mu_{i}$ and $\beta$. In the empirical section below, we will limit ourselves to two special cases, which we dub $\mathrm{A}^{1} \operatorname{MixStable}(k, g)$ and $\mathrm{A}^{2} \operatorname{MixStable}(k, g)$, respectively. In the former, we restrict $\beta$ to zero, whereas in the latter, $\mu_{i}=0$, so that only one source of asymmetry is considered at a time.

As the stable distribution does not possess a finite second moment if $\alpha<2$, the MixStable-GARCH process will not be covariance stationary. It may still be strictly stationary, however. This follows from Liu (2007) who generalizes the Markov-switching GARCH(1,1) process of Haas et al. (2004a) to allow for more general power parameters and innovation distributions, and studies its dynamic properties. This process nests the model studied in this paper, and we state the stationarity condition for the parsimonious case where all the stable components are characterized by the same shape parameters $\alpha$ and $\beta$. It then follows from Corollary 2.1 of Liu (2007) that a sufficient condition for the process to be strictly stationary with a finite $\delta$ th moment is that the eigenvalues of the matrix

$$
\gamma_{1} \boldsymbol{\omega}^{\prime} \kappa_{\delta, \alpha, \beta}+\Psi_{1}
$$

are inside the unit circle, where, as in Mittnik et al. (2002),

$$
\begin{aligned}
\kappa_{\delta, \alpha, \beta} & =\eta_{\delta}^{-1} \Gamma\left(1-\frac{\delta}{\alpha}\right)\left(1+\tau_{\alpha, \beta}^{2}\right)^{\delta /(2 \alpha)} \cos \left(\frac{\delta}{\alpha} \arctan \left(\tau_{\alpha, \beta}\right)\right), \\
\tau_{\alpha, \beta} & =\beta \tan (\alpha \pi / 2), \\
\eta_{\delta} & = \begin{cases}\Gamma(1-\delta) \cos \left(\frac{\pi \delta}{2}\right), & \text { if } \delta \neq 1, \\
\pi / 2, & \text { if } \delta=1 .\end{cases}
\end{aligned}
$$

Term $\kappa_{\delta, \alpha, \beta}$ is the power- $\delta$ absolute moment of a stable random variable with tail index $\alpha$ and asymmetry parameter $\beta$; see Paolella (2007, Sec. 8.3) for a detailed derivation. For $\delta=1$ and $\beta=0,(6)$ reduces to $\kappa_{1, \alpha, 0}=2 \Gamma\left(1-\alpha^{-1}\right) / \pi$.

Similar to the mixed normal GARCH model, condition (5) allows some (but not all) components to be driven by non-stationary GARCH dynamics, whereas overall the process will be stationary as long as the mixing weights of these components are sufficiently small. In particular, when $\max _{j}\left\{\psi_{j}\right\}<1$ is satisfied, it follows from arguments similar to Haas et al. (2004a) that the eigenvalue condition is equivalent to

$$
\sum_{j=1}^{k} \omega_{j} \frac{\kappa_{\delta, \alpha, \beta} \gamma_{1, j}}{1-\psi_{j}}<1
$$

The $\operatorname{ARCH}(\infty)$ representation of $\sigma_{j, t}^{\delta}$, given by

$$
\sigma_{j, t}^{\delta}=\frac{\omega_{j}}{1-\psi_{j}}+\gamma_{1, j} \sum_{i=1}^{\infty} \psi_{j}^{i-1}\left|\varepsilon_{t-i}\right|^{\delta}
$$


shows that the total impact of a shock on future volatility in regime $j$ is

$$
\gamma_{1, j} \sum_{i=0}^{\infty} \psi_{j}^{i}=\frac{\gamma_{1, j}}{1-\psi_{j}} .
$$

Thus, condition (7) restricts the average total impact of a shock on the component-specific future volatilities.

\section{Estimation of Mixture GARCH Models}

It is well-known (see Kiefer and Wolfowitz, 1956; and Day, 1969) that the likelihood function of a mixture is potholed with singularities (infinite likelihood values). In the estimation of MixNormal-GARCH models, such degenerated states can be avoided by using Bayesian estimation procedures as devised in Ausín and Galeano (2007) and Bauwens et al. (2007). A potential drawback of these approaches, however, is their computational complexity and relatively high estimation time, along with the added complexity of augmenting those procedures from the normal to the stable (or other) distribution. To address these shortcomings, we devise an augmented likelihood function which can still be maximized with conventional optimization techniques but, unlike the usual likelihood, completely and elegantly avoids degenerated mixture estimates. As the problem of avoiding degenerated mixture estimates is a general problem known by many names, we will refer to it as the mixture degeneracy problem.

\subsection{Augmented Likelihood Estimation}

Augmented likelihood estimation (ALE) is based on the observation that degenerated components yield zero likelihood values for all observations which differ from their location parameter. As such, singularities can be prevented by avoiding zero likelihood values in all mixture components. This idea is implemented in the augmented likelihood framework by adding the geometric average likelihood value of each mixture component to the overall likelihood function. The additional $k$ likelihood terms yield an infinite penalty whenever a mixture component degenerates. Thus, the ALE solves the mixture degeneracy problem without parameter constraints or penalty terms, but rather by shrinking the overall mixture likelihood function towards the component-wise likelihood functions.

Consider first estimation of an unconditional (no GARCH) mixture distribution. Let $\boldsymbol{\theta}=\left(\boldsymbol{\omega}, \boldsymbol{\theta}_{1}, \ldots, \boldsymbol{\theta}_{k}\right)^{\prime}$ denote the vector of model parameters, where $\boldsymbol{\omega}$ is the vector of mixture weights, and $\boldsymbol{\theta}_{i}$ represents the parameter vector of the density function, $f_{i}$, of the $i$ th component. Then, the ALE takes the form of $\hat{\boldsymbol{\theta}}_{\mathrm{ALE}}=\arg \max _{\boldsymbol{\theta}} \tilde{\boldsymbol{\ell}}(\boldsymbol{\theta} ; \boldsymbol{\varepsilon})$, where the augmented log-likelihood function $\tilde{\ell}$ consists of the usual sum of log-likelihood values $\ell^{\star}$, as well as the logarithmic geometric means of the component-wise likelihood series $\bar{\ell}_{i}$, i.e.,

$$
\begin{aligned}
\tilde{\ell}(\boldsymbol{\theta} ; \boldsymbol{\varepsilon}) & =\ell^{\star}(\boldsymbol{\theta} ; \boldsymbol{\varepsilon})+\sum_{i=1}^{k} \bar{\ell}_{i}\left(\boldsymbol{\theta}_{i} ; \boldsymbol{\varepsilon}\right) \\
& =\sum_{t=1}^{T} \log \sum_{i=1}^{k} \omega_{i} f_{i}\left(\varepsilon_{t} ; \boldsymbol{\theta}_{i}\right)+\sum_{i=1}^{k} \frac{1}{T} \sum_{t=1}^{T} \log f_{i}\left(\varepsilon_{t} ; \boldsymbol{\theta}_{i}\right) .
\end{aligned}
$$


Assuming (as we subsequently do) that the true mixture process is free of degenerated components and its standard likelihood function has a consistent root (compare Kiefer, 1978), the ALE is consistent, because, by dividing by $T$, the contribution to $\tilde{\ell}$ from the additional $k$ terms becomes negligible in the limit as $T \rightarrow \infty$ for all mixtures without degenerated components. ${ }^{3}$

\subsection{Augmented Mixture GARCH Estimation}

The augmented likelihood methodology above is tailored to unconditional mixtures and, thus, cannot prevent singularities in (time-) conditional mixture models. In $k$-component mixture GARCH models, for instance, singularities occur at all time points $t, t=1, \ldots, T$, and all components $i, i=1, \ldots, k$, where $\sigma_{i, t}=0$ and $\mu_{i}=\varepsilon_{t}$. We refer to this problem as the local degeneracy problem. Assuming that the true mixture GARCH process does not possess singular or near-singular components at any point in time, we devise two solutions for the local degeneracy problem based on the augmented likelihood methodology.

The first solution is built upon the ALE by removing singularities from the (mixture) likelihood function using a lower bound on the GARCH constant(s),

$$
\gamma_{i, 0}>c>0, \quad \text { for all } i=1, \ldots, k \text {. }
$$

This parallels the idea in Hathaway (1985) in the simple (unconditional) normal mixtures, and is extended in Tanaka (2009). Without local degeneracy, the ALE remains feasible and the mixture GARCH degeneracy problem can be solved as in the unconditional case. The restricted ALE (RALE) for the MixStable-GARCH model is given by $\hat{\boldsymbol{\theta}}_{\mathrm{RALE}}=\arg \max _{\boldsymbol{\theta}} \tilde{\ell}(\boldsymbol{\theta} ; \boldsymbol{\varepsilon})$ where (9) is satisfied and (8) becomes

$$
\tilde{\ell}_{S}(\boldsymbol{\theta} ; \boldsymbol{\varepsilon})=\sum_{t=1}^{T} \log \sum_{i=1}^{k} \omega_{i} f_{S}\left(\varepsilon_{t} ; \alpha_{i}, \beta_{i}, \mu_{i}, \sigma_{i, t}\right)+\sum_{i=1}^{k} \frac{1}{T} \sum_{t=1}^{T} \log f_{S}\left(\varepsilon_{t} ; \alpha_{i}, \beta_{i}, \mu_{i}, \sigma_{i, t}\right) .
$$

A shortcoming of this approach is the necessity of having to choose the tuning parameter $c$, and doing so in such a way that, as a function of sample size $T$, the sequence $c_{T}$ yields a consistent estimator. Extensive studies with the financial data used in this report, and with simulated data mimicking such finance data, yield that $c=0.01$ works well in the sense that, while (9) is occasionally binding, the parameter estimates barely differ from those obtained by the EALE method discussed next.

Our second solution to the local degeneracy problem is more general and can be applied to any conditional mixture model. Let $\ell \in \mathbb{R}^{T}$ denote a vector of (finite) log-likelihood values. Based on the observation that the sample geometric mean of the likelihood values

$$
\widehat{\mathbb{E}}_{\text {geo }}(\ell)=\exp \left\{\frac{1}{T} \sum_{t=1}^{T} \ell_{t}\right\}=\exp \{\bar{\ell}\}
$$

\footnotetext{
${ }^{3}$ An example of a non-consistent ALE (or MLE) can easily be constructed by imposing inappropriate parameter constraints. Indeed, this is the reason why the RALE (below) is not always a consistent estimator.
} 
grows at a slower rate than the sample variance-like quantity

$$
\widehat{\mathbb{V}}_{\text {geo }}(\ell)=\frac{1}{T} \sum_{t=1}^{T}\left(\exp \left\{\ell_{t}\right\}-\exp \{\bar{\ell}\}\right)^{2}
$$

if some likelihood values move towards infinity, i.e.,

$$
\frac{\widehat{\mathbb{E}}_{\text {geo }}(\ell)}{\widehat{\mathbb{V}}_{\text {geo }}(\ell)} \rightarrow 0, \text { if } \widehat{\mathbb{E}}_{\text {geo }}(\ell) \rightarrow \infty,
$$

we propose the component-wise penalty term, $\check{\ell}_{i}$,

$$
\check{\ell}_{i}\left(\boldsymbol{\theta}_{i} ; \boldsymbol{\varepsilon}\right)=\log \left(1+\frac{1}{T} \sum_{t=1}^{T}\left(\exp \left\{\ell_{i, t}\right\}-\exp \left\{\bar{\ell}_{i}\right\}\right)^{2}\right),
$$

where $\ell_{i, t}=\log f_{i}\left(\varepsilon_{t} ; \boldsymbol{\theta}_{i}\right)$. The component-wise incorporation of (10) into (8) consequently removes all local singularities. Moreover, as (10) is purely likelihood-based, the resulting estimator is also free of direct parameter constraints. The extended ALE (EALE) is given by $\hat{\boldsymbol{\theta}}_{\mathrm{EALE}}=\arg \max _{\boldsymbol{\theta}} \tilde{\tilde{\ell}}(\boldsymbol{\theta} ; \boldsymbol{\varepsilon})$ where

$$
\tilde{\tilde{\ell}}(\boldsymbol{\theta} ; \boldsymbol{\varepsilon})=\ell^{\star}(\boldsymbol{\theta} ; \boldsymbol{\varepsilon})+\sum_{i=1}^{k} \bar{\ell}_{i}\left(\boldsymbol{\theta}_{i} ; \boldsymbol{\varepsilon}\right)-\sum_{i=1}^{k} \check{\ell}\left(\boldsymbol{\theta}_{i} ; \boldsymbol{\varepsilon}\right) .
$$

Similar to the ALE, the additional terms vanish as $T \rightarrow \infty$, so that the EALE is consistent. The price to pay is that the penalty in (10) introduces a source of bias as the density function is penalized for (too) large likelihood values, thus implicitly transferring mass to the tail area. For the case of the MixStable-GARCH model, the expression specializes to

$$
\tilde{\tilde{\ell}}_{S}(\boldsymbol{\theta} ; \boldsymbol{\varepsilon})=\sum_{t=1}^{T} \log \left(\sum_{i=1}^{k} \omega_{i} L_{i, t}^{S}\right)+\sum_{i=1}^{k}\left\{\log g_{i}-\log \left(1+\frac{1}{T} \sum_{t=1}^{T}\left(L_{i, t}^{S}-g_{i}\right)^{2}\right)\right\}
$$

where $L_{i, t}^{S}=f_{S}\left(\varepsilon_{t} ; \alpha_{i}, \beta_{i}, \mu_{i}, \sigma_{i, t}\right)$ and $g_{i}=\left(\prod_{t=1}^{T} L_{i, t}^{S}\right)^{1 / T}$.

Both RALE and EALE yielded essentially the same maximized likelihood values in all runs we conducted based on real and simulated data. (It is important to emphasize that the actual, and not the augmented, likelihood is being referred to here.) While there was a slight preference for the EALE over the RALE in terms of actual likelihood value, both resulted in essentially identical out-of-sample forecasts. The local degeneracy problem for MLE, RALE and EALE is further illustrated in Figure 1. For all data generating processes we have studied, the results look qualitatively the same: while RALE and EALE never yield degenerated estimates (all likelihood values are finite), the standard MLE frequently results in degenerated estimates (infinite likelihood values). It is due to the imperfection of global optimization methods that, first, the MLE does not always degenerate, and second, that degenerated estimates occur less frequently for larger sample sizes, as can be seen by comparing the left and right panels in Figure 1. 


\subsection{Small Sample Properties}

Simulation studies were conducted in order to assess the distributional properties of the estimators, partly to compensate for the fact that the asymptotic properties are elusive at present. The parameters of the data generating processes we consider are calibrated to actual finance data. The first analysis is based on the estimation error $\epsilon_{i, j}^{P}=\hat{\theta}_{i, j}-\theta_{j}, i=1, \ldots, N$, $j=1, \ldots, M$, where $\boldsymbol{\theta}$ is the parameter vector of the true process, $M$ is the total number of parameters, and $N=1000$ the number of simulations. To address the label switching problem, parameter vectors are sorted by mixture weights. Table 1 shows the results of two simulation studies for the MixStable $(k, g)$ model. ${ }^{4}$ Two measures are reported. The first is the usual root mean squared error of the parameters (P-RMSE), $\sqrt{\sum_{i}\left(\epsilon_{i, j}^{P}\right)^{2} / N} \cdot{ }^{5}$ The second measure is the inter-quantile range (IQR) ${ }^{6}$ As expected, all error measures decrease as the sample size increases.

We now turn to the second analysis. The above parameter error diagnostic needs to be augmented as a basis for the analysis for two reasons: First, mixture processes can often be mimicked quite accurately by processes with fairly different parameter vectors. Second, the sorting of mixture components may lead to false results, e.g., if some of the true mixture weights are close. Hence, we consider two proxy measures that reflect the main characteristics of mixture GARCH processes. The first is based on the quantiles of the unconditional distribution of the process and the second uses the autocorrelation function (acf) of the absolute returns. (In order to compare the sample values to their theoretical counterparts, the latter are obtained via simulation of the true process using one million observations.) The corresponding RMSE measures (Q- and A-RMSE) are given by the square root of the mean squared

$$
\epsilon_{i, j}^{Q}=\text { quantiles }(\hat{\theta}, \boldsymbol{\zeta})_{i, j}-\text { quantiles }(\theta, \boldsymbol{\zeta})_{0, j} \text { and } \epsilon_{i, j}^{A}=\operatorname{acf}(\hat{\theta}, \rho)_{i, j}-\operatorname{acf}(\theta, \rho)_{0, j},
$$

respectively, where we evaluate the sample quantile function at the probability levels $\boldsymbol{\zeta}=$ $(0.01,0.1,0.2, \ldots, 0.9,0.99)$ and compute the sample acf with lag order $\rho=100$.

For comparison we also report the performance of the standard MLE (although it is ill-defined for mixtures). As expected, the MLE often results in estimates for which at least one component explains only a few observations. Such mixture components are typically either degenerated or correspond to local optima where fewer mixture components explain the data (in the sense of almost zero-valued mixture weights) than actually being estimated. Clearly, RALE and EALE outperform the MLE here, but also suffer from (non-singular) local optima, though substantially less. Similarly, it is evident that the EALE results in

\footnotetext{
${ }^{4}$ More detailed results and information on computational aspects are available from the authors upon request.

${ }^{5}$ We exclude those runs (estimated parameter vectors) such that one or more components had an excessively small weight (in particular, when $\omega_{i} T<10$, e.g., the $i$ th component explains less than $0.1 \%$ of the observations when using $T=1000)$. We do this to account for the RMSE sensitivity to numerical outliers in the parameter estimates of such components.

${ }^{6}$ Unlike the P-RMSE, the IQR is based on all runs, as it is not affected by outliers.
} 
non-stationary estimates more often than the RALE. The reason is that in order to match a given unconditional volatility, an increase in the GARCH constant must be offset by a decrease in the remaining GARCH parameters, thus moving them away from the nonstationarity border. This shortcoming of the EALE, however, vanishes as the sample size increases. In contrast, by looking at the IQR values based on all estimates, Table 1 indicates that RALE and EALE perform very similar; in the two-component case the RALE slightly outperforms the EALE, while it is the other way around in the three-component case. Although the RALE tends to be slightly faster, we opt for the EALE in the following, as it has the appealing advantage of avoiding direct parameter constraints and is therefore an unrestricted solution of the mixture degeneracy problem. ${ }^{7}$

\section{Univariate Empirical Results}

Our empirical analysis covers the major international equity indices DAX 30, S\&P 500, DJIA 30, NIKKEI 225 and NASDAQ COMPOSITE (20 years, dating back from July 7th, 2009; resulting in a sample size of 2609) as well as the exchange rates JPY/EUR and USD/EUR (10 years, dating back from July 7th, 2009; resulting in a sample size of 1304). All results are based on percentage $\log$ returns, $\varepsilon_{t}=100\left(\log p_{t}-\log p_{t-1}\right)$, where $p_{t}$ is the daily closing index price at time $t$. We study $\operatorname{MixNormal}(k, g), \operatorname{MixGED}(k, g)$ and MixStable $(k, g)$ models, where $(k, g) \in\{(2,2),(3,2),(3,3),(4,4)\} .{ }^{8}$ To prevent overfitting, shape parameters are restricted to be identical. ${ }^{9}$

Cutting to the chase, the best performing models (in terms of both in-sample fit and, more importantly, out-of-sample performance) are all in the MixStable class. In-sample, the $\mathrm{A}^{2}$ MixStable $(2,2)$ model is favored by the BIC, while out-of-sample, the $\mathrm{A}^{2}$ MixStable $(2,2)$, as well as the $\mathrm{A}^{2}$ MixStable(3,2) and $\mathrm{A}^{2}$ MixStable(3,3), perform well. It is noteworthy that the $\mathrm{A}^{1}$ MixStable $(4,4)$ model, despite its high parametrization, performs overall best in terms of the uniformity of the predictive cdf values. Nevertheless, the $\mathrm{A}^{2}$ MixStable $(3,2)$ model might be preferred because of its relatively parsimonious parametrization (which also

\footnotetext{
${ }^{7}$ Regarding computation time: On an Intel i7-2600K quad-core processor at $4.2 \mathrm{Ghz}$ a single estimation (EALE) of the MixStable(4,4) model based on 1000 data points (running in Matlab R2010a on a single core of the cpu) takes up to four minutes using Nolan's fast spline approximation and about four times the amount of time using the vectorized version of Zolotarev's integral expression. See WP for further details regarding computation time and differences in accuracy in these two methods.

${ }^{8}$ Several models with $k>4$ were also considered; information criteria never favored them, out-of-sample forecasts were roughly comparable to the $k=4$ case but increasingly mixed for higher $k$, and estimations resulted more frequently in estimates with mixture weights close to zero for the additional components. We do not report the results.

${ }^{9}$ By relaxing the equality of the shape parameters constraint, the resulting estimates show an undesired property: While very few mixture components explain the majority of the data with reasonable parameter estimates, the majority of the mixture components maximizes the likelihood based on corner solutions for their shape parameters, which is a classic indicator of overfitting. Using 1000 observations, the $\operatorname{MixGED}(k, g)$ and $\operatorname{MixStable}(k, g)$ are frequently overfitted, while for larger data sets, the overfitting vanishes, as would be expected, and for the full DJIA sample, $\operatorname{MixGED}(k, g)$ estimates with free shape parameters are found to be in line with those reported in Rombouts and Bouaddi (2009).
} 
implies faster estimation). It also performed slightly better than the $\mathrm{A}^{1} \operatorname{MixStable}(4,4)$ for the VaR comparisons at the lower probability levels.

\subsection{Choice of $\delta$}

We investigate the influence of the GARCH power parameter $\delta$, for which $\delta<\alpha$ must be satisfied in order to ensure the existence of the MixStable-GARCH process. In particular, given the flexibility and richness of the model, we have confirmed for numerous data sets and sample sizes that, relative to the other parameters, the likelihood is relatively flat in $\delta$. This can lead to exacerbated estimation problems (in addition to requiring dynamically imposed constraints, as opposed to simple box constraints, during estimation), and implies that just setting $\delta$ to a compromise value will not lead to appreciably poorer (and could possibly lead to slightly better) forecasts. We find that the choice of $\delta=1$ is not only adequate, but also conveniently satisfies the $\delta<\alpha$ constraint. Details for this conclusion, based on the use of the profile log likelihood function for in-sample analysis, and on the results for out-of-sample forecast comparisons, can be found in WP. Observe that setting $\delta=1$ implies that the MixStable-GARCH model no longer nests the MixNormal $(k, g)$ model in (1) and (2), but rather a variant of it, in which the exponent of 2 is replaced by 1 .

\subsection{In-Sample Fit}

For assessing in-sample properties, we fit the MixNormal-, MixGED- and MixStableGARCH models to the seven financial return series under study using the extended likelihood estimator (EALE) from Section 3.2. (As an illustration, parameter estimates of the two MixStable-GARCH models for the DJIA return data are given in WP.)

Table 2 shows the BIC measure of all models and data sets under study (the AIC is reported in WP). We focus on the BIC because the literature on mixture models provides some theoretical and empirical support for its appropriateness and good performance, in particular for selecting the number of mixture components (see, e.g., Keribin, 2000; Francq et al., 2001; and Frühwirth-Schnatter, 2006, Ch. 4). The BIC, which favors less densely parametrized models, has an overall tendency towards the $\mathrm{A}^{2} \operatorname{MixStable}(2,2)$ model; the AIC shows a less clear pattern.

\subsection{Comparison of Forecasting Performance}

While a model as flexible as the MixStable $(k, g)$ should be expected to provide an excellent in-sample fit to virtually any return series compared with more traditional GARCH-type models, the concern remains as to whether the relatively large parametrization, the nontrivial computational aspects of the stable density, and the degeneracy issue associated with mixtures warrant its use. To judge this, we compare the empirical performance of the onestep-ahead predictive cdfs across models using tests for uniformity (see below) as well as concentrating on the left tail, using probability values typical for VaR calculations.

The choice of the window size is a tuning parameter chosen to maximize the quality of future risk or density forecasts of the particular set of assets under study. It is not necessarily the case that more observations are better, because, with certainty, the proposed model differs in some way from the true data generating process, which itself is likely to 
not be strictly stationary over long periods of time. A model is possibly, however, a good approximation to reality for short periods of time, though using too short a window results in high variance of the parameters and thus inferior forecasts. To negotiate this bias/variance tradeoff, some experiments with the MixStable-GARCH model and two of the data sets under study indicate that use of a rolling window of sample size 1000 is superior to use of either 500 or 2000 . While this value could be optimized further by conducting dedicated experiments per dataset, we use 1000 in all of the following.

For all models considered, we re-estimate the model parameters every 20 trading days (about once a month), so that each estimation contains $2 \%$ of new data. Our analysis is based on the realized predictive cdf values obtained from evaluating the one-step-ahead cdf forecasts at the realized returns. If the model is correct, it is well-known that these are uniformly distributed.

Let $\hat{p}_{t}=\hat{F}_{t \mid \mathcal{F}_{t-1}}\left(\varepsilon_{t} ; \hat{\boldsymbol{\theta}}_{t-h}\right), t=1, \ldots, N$, be the sequence of realized predictive cdf values, noting that, for each $t$, the parameter vector is estimated using information (in this case, just the past returns) up to and including time $t-h$, where $h$ is a value in $\{1,2, \ldots, 20\}$, but the entire return series up to time $t-1$ is used in the model filter. Finally, this predictive cdf is evaluated at the actual return at time $t$. Denote the collection of these $N$ values as vector $\hat{\boldsymbol{p}}$. Further let $\hat{\boldsymbol{p}}^{[s]}$ denote the sorted vector, $\hat{p}_{1}^{[s]} \leq \hat{p}_{2}^{[s]} \leq \cdots \leq \hat{p}_{N}^{[s]}$. The Anderson-Darling (AD) and Cramér-von Mises (CM) test statistics are given respectively by

$$
\mathrm{AD}=-N-\sum_{i=1}^{N} \frac{2 i-1}{N}\left(\log \left(\hat{p}_{i}^{[s]}\right)+\log \left(1-\hat{p}_{N-i+1}^{[s]}\right)\right)
$$

and

$$
\mathrm{CM}=\frac{1}{12 N}+\sum_{i=1}^{N}\left(\frac{2 i-1}{2 N}-\hat{p}_{i}^{[s]}\right)^{2} .
$$

In addition, we provide test statistics for the Kolmogorov-Smirnov (KS) test for uniformity, as well as the Jarque-Bera (JB) and Shapiro-Wilk (SW) tests for normality after applying the inverse normal cdf transform. We test for serial correlation in $\hat{\boldsymbol{p}}$ (as a proxy for the iid property) and report Ljung-Box (LB) test statistics,

$$
\mathrm{LB}=N(N+2) \sum_{i=1}^{m} \frac{\hat{\rho}_{i}^{2}}{N-i},
$$

where $\hat{\rho}_{i}$ is the $i$ th autocorrelation from the $i$ th sample autocorrelation function.

Tables 3 and 4 show the results. It is important to note that here, we are testing the prediction quality over the whole support of the distribution, and not just the left tail (as we do below, for directly testing the quality of value at risk predictions). Except for DAX and NASDAQ, AD and CM are clearly in favor of the stable models with a strong preference for the $\mathrm{A}^{1}$ MixStable-GARCH model. Results for KS and LB are less clear but also pro stable in four out of seven cases. Similar results are obtained for both normality tests, though the JB test appears to favor the $\mathrm{A}^{2}$ MixStable-GARCH model. 
We also consider VaR measures dedicated to the left tail, as these are of possibly even greater interest from a risk management perspective. Table 5 shows the empirical coverage probabilities (as percentages) for the 1\% and 5\% VaR levels along with $p$-values indicating the severeness of potential risk underestimation. The results for $5 \%$ are mixed, though at the 1\% VaR level (arguably the most important VaR level in risk management applications), the $\mathrm{A}^{2}$ MixStable $(k, g)$ model clearly outperforms the other models for most data sets under study.

For further investigations of the VaR prediction quality, we adopt a simple quality measure based on the coverage error over the VaR levels up to $100 \lambda \%$, see Kuester et al. (2006). The measure calculates the deviation between predictive cdf and uniform cdf and, thus, captures the excess of percentage violations over the VaR levels, where the deviation is defined as $100\left(F_{U}-\hat{F}_{e}\right)$ with $F_{U}$ being the cdf of the standard uniform random variable and $\hat{F}_{e}$ referring to the empirical cdf formed from $\hat{\boldsymbol{p}}$. Building upon this metric we report the integrated root mean squared error (IRMSE) over the left tail up to the maximal VaR level of interest. The IRMSE employed herein is closely related to the CM statistic but with the sum truncated at $h=\lceil\lambda N\rceil$, i.e.,

$$
\operatorname{IRMSE}=\sqrt{\frac{1}{h} \sum_{i=1}^{h}\left(100 \frac{2 i-1}{2 N}-100 \hat{p}_{i}^{[s]}\right)^{2}} .
$$

The results in Table 6 confirm the superiority of the stable models in five out of seven cases at the $1 \%$ level.

Finally, we investigate the hit sequence of realized predictive VaR violations,

$$
v_{t}=\mathbf{1}_{\varepsilon_{t} \leq \hat{q}_{t}}, \quad \hat{q}_{t}=\widehat{\mathrm{VaR}}_{t \mid \mathcal{F}_{t-1}}(\lambda)
$$

where $\mathbf{1}$ is the indicator function. Under the null of correct conditional coverage, the $v_{i}$ are iid Bernoulli $(\lambda)$. From this sequence, the test statistic $L R_{C C}=L R_{U C}+L_{I N D}$ is computed, as proposed in Christoffersen (1998), where $L R_{U C}$ and $L R_{I N D}$ test for unconditional coverage and independence, respectively. As can be seen from Table 7 for the $1 \%$ VaR level, the tendency of $\operatorname{MixStable}(k, g)$ (in particular of $\mathrm{A}^{2}$ MixStable-GARCH) to outperform $\operatorname{MixNormal}(k, g)$ and $\operatorname{MixGED}(k, g)$ is also corroborated by the Christoffersen test.

\section{ICA-MixStable-GARCH}

A direct generalization of the MixNormal-GARCH model to the multivariate setting with $D$ assets has been investigated by Bauwens et al. (2007) and Haas et al. (2009), the latter model allowing for asymmetries. While of value for a small number of assets, those models will not be practical for even modest portfolios, let alone large ones. In addition, attempting to extend that model to support the multivariate stable distribution is not trivial (but see Lombardi and Veredas, 2009; Bonato, 2011; and the references therein).

A multivariate distribution with mix-Stable GARCH marginals can be constructed via use of independent components analysis (ICA); see, e.g., Hyvärinen et al. (2001). Crucially, 
the resulting multivariate distribution is such that the distribution of a linear combination (as needed to conduct portfolio optimization) is tractable. The method assumes a set of nonGaussian distributed independent random variables of which linear combinations in the form of time series have been observed. The goal is to recover the original independent random vectors of time series, called the independent components. Once the mixing matrix is known (estimated), the independent components can be modeled and forecasted independently (by, for example, a GARCH-type model). The related concept of conditionally uncorrelated components is discussed in Fan et al. (2008).

An application of the popular iterative FastICA algorithm can be found in Broda and Paolella (2009), where the method is used in a portfolio allocation exercise to estimate the independent components (driven by generalized hyperbolic innovations) of the 30 constituents of the Dow Jones Industrial Average index. To be precise, the ICA variant employed therein maximizes the conditional heteroskedasticity of the independent components. We refer to this specific version as CHICA (Conditionally Heteroskedastic ICA). Unfortunately, the CHICA method has the drawback of requiring finite fourth moments, which is not fulfilled in our setting. However, to the best of our knowledge, the only ICA method dedicated to (time-correlated) stable driven data is found in Fabricius et al. (2001). It is all the more remarkable that in our extensive simulation studies, the method is outperformed by the CHICA method.

Briefly, the CHICA method is a two-step procedure, separating the estimation of the correlation structure from that of the univariate dynamics; details are given in Chen et al. (2006) and Broda and Paolella (2009). A first step is to estimate the expectation of the $D$ assets and obtain the matrix of de-meaned returns $\boldsymbol{Y} \in \mathbb{R}^{D \times T}$. There are several ways of doing this. One could just use the sample mean for each series, though given the fat-tailed nature of the data, this is not efficient. A trimmed mean, or even the median, might be superior, or perhaps best, use of the location term jointly estimated with, say, a MixStableGARCH model. Whichever is used, further improvement might be realized by (i) estimating it using weighted likelihood, with relatively more weight on more recent observations (see Paolella and Steude, 2008; Broda and Paolella, 2011; and the references therein) and/or (ii) shrinking the $D$ values towards, say, zero.

In the second step, the CHICA method is applied to the residual series $\boldsymbol{Y}$ estimating the mixing matrix $\boldsymbol{A} \in \mathbb{R}^{D \times D}$ that best separates the signals maximizing their GARCH effects. Given the mixing matrix $\boldsymbol{A}$, the $D$ independent components, $\boldsymbol{X} \in \mathbb{R}^{D \times T}$, are obtained by $\boldsymbol{X}=\boldsymbol{A}^{-1} \boldsymbol{Y}$. The third (and only time-consuming) step consists in the estimation and prediction of the $D$ (univariate) independent components based on the MixStable-GARCH model. Observe that the three above steps (in particular, the last one) do not need to be repeated for different portfolio weights. This is crucial if such weights are to be optimized. Note also that each of the $D$ components is endowed with its own, estimated, MixStableGARCH model, and so each has its own tail index $\alpha$. This can be contrasted with attempts using the multivariate stable distribution, which has only a single $\alpha$ for all assets.

Suppressing the time index for readability, the return $R$ on a portfolio of $D$ assets is a weighted sum of the individual asset returns, and consequently (via the matrix A) also a weighted sum of the independent components $X_{j}$ with associated weights $b_{j}$, say. The 
distribution of this weighted sum can be computed from its characteristic function (cf), which, from independence, factors into the product of the cfs of the individual components. In our context, each $X_{j}$ is a $k$-component stable mixture, with pdf given by $f_{X_{j}}(x)=$ $\sum_{i=1}^{k} \omega_{j, i} f_{S}\left(x ; \alpha_{j, i}, \beta_{j, i}, \mu_{j, i}, \sigma_{j, i}\right)$. As the of of a stable random variable with tail index $\alpha$, asymmetry parameter $\beta$, location $\mu$ and scale $\sigma$ is

$$
\exp \left\{\imath \mu t-\sigma^{\alpha}|t|^{\alpha}(1-\imath \beta \operatorname{sign}(t) \tan (\alpha \pi / 2))\right\},
$$

$\imath$ denoting the imaginary unit, the cf of $X_{j}$ is given by

$$
\varphi_{X_{j}}(t)=\sum_{i=1}^{k} \omega_{j, i} \exp \left\{\imath \mu_{j, i} t-\sigma_{j, i}^{\alpha_{j, i}}|t|^{\alpha_{j, i}}\left(1-\imath \beta_{j, i} \operatorname{sign}(t) \tan \left(\alpha_{j, i} \frac{\pi}{2}\right)\right)\right\},
$$

from which a simple expression for $\varphi_{R}(t)$ follows. Standard inversion methods can then be applied to $\varphi_{R}$ to calculate its pdf or cdf; see, e.g., Paolella (2007, Chapter 1) for details.

For MixStable $(k, g)$, the model lends itself to the computation of portfolio risk measures such as expected shortfall (ES) or VaR due to its simple of expression. (Interestingly, as shown in Sy (2006), the VaR is a coherent risk measure for portfolios of independent stable Paretian distributed assets, but only if the tail exponent $\alpha$ is the same for all assets.) For continuous return $R$, the (relative) expected shortfall at $100 \lambda \%$ level, $0<\lambda<1$, is

$$
\mathrm{ES}_{R}^{\lambda}=-\mathbb{E}[R \mid R<q]=-\frac{1}{\lambda} \mathbb{E}\left[R \mathbf{1}_{R<q}\right]
$$

where $\mathbf{1}$ is the indicator function and $q=-\operatorname{VaR}_{R}(\lambda)$, the $100 \lambda \%$ quantile.

If $R$ were just a mixture of stable distributions, one could compute $\mathbb{E}\left[R \mathbf{1}_{R<q}\right]$ via the real integral expression for the stable ES in Stoyanov et al. (2006) combined with the general results for the ES of mixtures in Broda and Paolella (2011). However, as $R$ is a convolution of mixtures of stables, this is not applicable. Neither is the general result of Kim et al. (2009), which requires that the cf be finite in a strip containing the real axis, which is not the case for stable distributions and their mixtures. To compute $\mathbb{E}\left[R \mathbf{1}_{R<q}\right]$, we use the result from Broda (2011) which applies more generally than that given in Kim et al. (2009),

$$
\mathbb{E}\left[R \mathbf{1}_{R<q}\right]=\frac{\varphi_{R}^{\prime}(0)}{2 \imath}+\frac{1}{\pi} \int_{0}^{\infty} \operatorname{Re}\left[\mathrm{e}^{-\imath t q} \varphi_{R}^{\prime}(t)\right] \frac{\mathrm{d} t}{t},
$$

where $\varphi_{R}^{\prime}(s)$ is the first derivative of the characteristic function of $R$, as derived in Appendix A.

Taken together, the computational ingredients are now available to do portfolio optimization in return/ES space, using ICA with the independent components modeled as MixStable processes. The resulting portfolio will, by necessity, have Pareto-like tails, no matter how many assets are involved, unlike in Broda and Paolella (2009), for which, as it uses the normal inverse Gaussian distribution for the components, eventually as $D$ increases, a central limit theorem effect will kick in, and the results will not differ from using a standard Markowitz approach. Given the number of tuning parameters and dependence on the choice of data, the assessment of the performance of the proposed method for portfolio allocation deserves a separate study, and is not pursued here. 


\section{Conclusions and Future Research}

An open question in financial econometrics is the suitability of infinite-variance distributions for modeling the unconditional or conditional distribution of asset returns. As discussed at length in the introduction, there is an ongoing debate regarding the maximally existing moment of asset returns. In this paper, we take the stance, as argued by numerous researchers in the stable Paretian field, that the stable Paretian distribution has many positive characteristics for modeling asset returns that outweigh its potential shortcomings. Indeed, we find that a model incorporating mixtures, GARCH, and underlying stable distributions yields a complex but coherent and statistically well motivated model which, based on extensive empirical exercises, delivers both excellent in-sample fit and, most relevantly, admirable out-of-sample forecasting results. In particular, the $\mathrm{A}^{2} \operatorname{MixStable}(3,2)$, $\mathrm{A}^{2}$ MixStable $(3,3)$ and $\mathrm{A}^{1}$ MixStable $(4,4)$ models performed best out-of-sample.

Various model extensions suggest themselves for future consideration. There could be value in generalizing the law of motion for the scale terms along the lines of the asymmetric GARCH models in Ding et al. (1993) or Alexander and Lazar (2009). The incorporation of a Markov switching structure, as done in Haas et al. (2004a) and Bauwens et al. (2010), or extension to time-varying component weights, as recently investigated by Bauwens and Storti (2009), could also (and possibly in addition) be entertained.

\section{Acknowledgements}

The authors are grateful to Kerstin Kehrle, Mico Loretan, Benoît Mandelbrot, J. Huston McCulloch, John P. Nolan, Eric Renault, Gennady Samorodnitsky, Murad S. Taqqu, David Veredas, Casper G. de Vries, and two anonymous referees, for providing an array of insightful and detailed comments and suggestions which led to a greatly improved paper. Part of the research of Paolella has been carried out within the National Centre of Competence in Research "Financial Valuation and Risk Management" (NCCR FINRISK), which is a research program supported by the Swiss National Science Foundation.

\section{Appendix}

\section{A. First Derivative of the Characteristic Function of a Weighted Sum of Mixed Stable Random Variables}

For evaluating the ES of a portfolio of mixed stable distributed asset returns based on (12), we derive the first derivative of the of of sum of mixtures of stable Paretian random variables in the general case of asymmetric stable random variables and unrestricted portfolio weights. Similar to the portfolio of $D$ assets in Section 5 , with summation weights $\boldsymbol{b} \in \mathbb{R}^{D}$, the portfolio sum is given by $R=\sum_{j=1}^{D} b_{j} X_{j}$, where the $X_{j}$ are independent $k$-component mixtures of stable random variables,

$$
X_{j} \sim \operatorname{MixStable}\left(\boldsymbol{\omega}_{j}, \boldsymbol{\alpha}_{j}, \boldsymbol{\beta}_{j}, \boldsymbol{\mu}_{j}, \boldsymbol{\sigma}_{j}\right), \quad j=1, \ldots, D
$$


with mixture weights $\boldsymbol{\omega}_{j} \in(0,1)^{k}, \sum_{i} \omega_{j, i}=1$, tail indices $\boldsymbol{\alpha}_{j} \in(0,2]^{k}$, skewness coefficients $\boldsymbol{\beta}_{j} \in[-1,1]^{k}$, location parameters $\boldsymbol{\mu}_{j} \in \mathbb{R}^{k}$ and scale terms $\boldsymbol{\sigma}_{j} \in \mathbb{R}_{>0}^{k}$. The cf of $R$ is straightforward to calculate and takes the form of

$$
\varphi_{R}(t ; \boldsymbol{\omega}, \boldsymbol{b}, \boldsymbol{\alpha}, \boldsymbol{\beta}, \boldsymbol{\mu}, \boldsymbol{\sigma})=\prod_{j=1}^{D} \sum_{i=1}^{k} \omega_{j, i} \varphi_{P}\left(t ; b_{j}, \alpha_{j, i}, \beta_{j, i}, \mu_{j, i}, \sigma_{j, i}\right),
$$

where

$\varphi_{P}(t ; b, \alpha, \beta, \mu, \sigma)=\varphi_{W}(t ; \alpha, \beta, b \mu, b \sigma)=\exp \left\{-|\sigma b t|^{\alpha}\left(1-\imath \beta \operatorname{sgn}(b t) \tan \left(\alpha \frac{\pi}{2}\right)\right)+\imath \mu b t\right\}$,

with $W(\cdot, \alpha, \beta, \mu, \sigma)$ being a stable Paretian random variable with tail index $\alpha$, asymmetry $\beta$, location $\mu$, and scale $\sigma$. Straightforward but tedious algebra shows that the first derivative of (A.1) is

$$
\varphi_{R}^{\prime}(t ; \boldsymbol{\omega}, \boldsymbol{b}, \boldsymbol{\alpha}, \boldsymbol{\beta}, \boldsymbol{\mu}, \boldsymbol{\sigma})=\sum_{i=1}^{D}\left(s_{i}^{\prime}+\sum_{j=1, j \neq i}^{D} s_{j}\right)
$$

where

$$
\begin{aligned}
& s_{j}=\sum_{i=1}^{k} \omega_{j, i} \varphi_{P}\left(t ; b_{j}, \alpha_{j, i}, \beta_{j, i}, \mu_{j, i}, \sigma_{j, i}\right), \quad j=1, \ldots, D, \\
& s_{j}^{\prime}=\sum_{i=1}^{k} \omega_{j, i} \varphi_{P}^{\prime}\left(t ; b_{j}, \alpha_{j, i}, \beta_{j, i}, \mu_{j, i}, \sigma_{j, i}\right), \quad j=1, \ldots, D,
\end{aligned}
$$

and, for $t \neq 0$,

$$
\begin{aligned}
\varphi_{P}^{\prime}(t ; b, \alpha, \beta, \mu, \sigma) & =\frac{\partial}{\partial t} \varphi_{P}(t ; b, \alpha, \beta, \mu, \sigma) \\
& =\varphi_{P}(t ; b, \alpha, \beta, \mu, \sigma)\left(\frac{\alpha}{t} \nu+\imath \mu b\right)
\end{aligned}
$$

with

$$
\nu=-|\sigma b t|^{\alpha}\left(1-\imath \beta \operatorname{sgn}(b t) \tan \left(\alpha \frac{\pi}{2}\right)\right)
$$

\section{References}

Adler, R. J., 1997. Discussion: Heavy Tail Modeling and Teletraffic Data. The Annals of Statistics 25 (5), 1849-1852.

Alexander, C., Lazar, E., 2006. Normal Mixture GARCH(1,1): Applications to Exchange Rate Modelling. Journal of Applied Econometrics 21, 307-336.

Alexander, C., Lazar, E., December 2009. Modelling Regime-Specific Stock Price Volatility. Oxford Bulletin of Economics and Statistics 71 (6), 761-797. 
Ausín, M. C., Galeano, P., 2007. Bayesian estimation of the Gaussian mixture GARCH model. Computational Statistics \& Data Analysis 51 (5), 2636-2652.

Bai, X., Russell, J. R., Tiao, G. C., 2003. Kurtosis of GARCH and Stochastic Volatility Models with Non-Normal Innovations. Journal of Econometrics 114, 349-360.

Bauwens, L., Hafner, C. M., Rombouts, J. V. K., 2007. Multivariate Mixed Normal Conditional Heteroskedasticity. Computational Statistics \& Data Analysis 51 (7), 3551-3566.

Bauwens, L., Preminger, A., Rombouts, J., 2010. Theory and inference for a Markov switching GARCH model. Econometrics Journal 13 (2), 218-244.

Bauwens, L., Storti, G., 2009. A Component GARCH Model with Time Varying Weights. Studies in Nonlinear Dynamics \& Econometrics 13 (2), Article 1.

Bollerslev, T., 1986. Generalized Autoregressive Conditional Heteroskedasticity. Journal of Econometrics 31, 307-327.

Bonato, M., 2011. Modeling fat tails in stock returns: a multivariate stable-GARCH approach. Computational Statistics 26, 1-23.

Broda, S. A., 2011. Tail Probabilities and Partial Moments for Quadratic Forms in Multivariate Generalized Hyperbolic Random Vectors, working Paper, University of Amsterdam.

Broda, S. A., Haas, M., Krause, J., Paolella, M. S., Steude, S. C., 2011. Stable Mixture GARCH Models. Swiss Finance Institute (SFI) Research Paper Series.

Broda, S. A., Paolella, M. S., 2009. CHICAGO: A Fast and Accurate Method for Portfolio Risk Calculation. Journal of Financial Econometrics 7 (4), 412-436.

Broda, S. A., Paolella, M. S., 2011. Expected Shortfall for Distributions in Finance. In: Čížek, P., Härdle, W., Rafał Weron (Eds.), Statistical Tools for Finance and Insurance.

Chen, Y., Härdle, W., Spokoiny, V., 2006. GHICA — Risk Analysis with GH Distributions and Independent Components, sFB 649 Discussion Paper 2006-78, Humboldt-University, Berlin.

Christoffersen, P. F., 1998. Evaluating Interval Forecasts. International Economic Review 39 (4), $841-862$.

Day, N. E., 1969. Estimating the Components of a Mixture of Normal Distributions. Biometrika 56 (3), $463-474$.

Ding, Z., Granger, C. W. J., Engle, R. F., 1993. A Long Memory Property of Stock Market Returns and a New Model. Journal of Empirical Finance 1, 83-106.

Doganoglu, T., Hartz, C., Mittnik, S., 2007. Portfolio Optimization when Risk Factors are Conditionally Varying and Heavy Tailed. Computational Economics 29 (3-4), 333-354.

Fabricius, T., Kidmose, P., Hansen, L., 2001. Dynamic components of linear stable mixtures from fractional low order moments. Acoustics, Speech, and Signal Processing, IEEE International Conference on 6, 39573960.

Fama, E. F., 1965. The Behavior of Stock Market Prices. Journal of Business 38, 34-105.

Fan, J., Wang, M., Yao, Q., May 2008. Modelling multivariate volatilities via conditionally uncorrelated components. Journal of the Royal Statistical Society: Series B (Statistical Methodology) 70, 679-702.

Francq, C., Roussignol, M., Zakoïan, J.-M., March 2001. Conditional Heteroskedasticity Driven by Hidden Markov Chains. Journal of Time Series Analysis 22 (2), 197-220.

Frühwirth-Schnatter, S., 2006. Finite Mixture and Markov Switching Models. Springer, New York.

Giacometti, R., Bertocchi, M. I., Rachev, S. T., Fabozzi, F. J., 2007. Stable Distributions in the BlackLitterman Approach to Asset Allocation. Quantitative Finance 7 (4), 423-433.

Haas, M., Mittnik, S., Paolella, M. S., 2004a. A New Approach to Markov Switching GARCH Models. Journal of Financial Econometrics 2 (4), 493-530.

Haas, M., Mittnik, S., Paolella, M. S., 2004b. Mixed Normal Conditional Heteroskedasticity. Journal of Financial Econometrics 2 (2), 211-250.

Haas, M., Mittnik, S., Paolella, M. S., 2009. Asymmetric Multivariate Normal Mixture GARCH. Computational Statistics \& Data Analysis 53 (6), 2129-2154.

Haas, M., Paolella, M. S., 2011. Mixture and Regime-switching GARCH Models. In: Bauwens, L., Hafner, C., Laurent, S. (Eds.), Handbook of Volatility Models and Their Applications. Wiley.

Hathaway, R. J., 1985. A Constrained Formulation of Maximum-Likelihood Estimation for Normal Mixture 
Distributions. The Annals of Statistics 13 (2), 795-800.

Hill, B. M., 1975. A Simple General Approach to Inference About the Tail of a Distribution. Annals of Statistics 3 (5), 1163-1174.

Hyvärinen, A., Karhunen, J., Oja, E., May 2001. Independent Component Analysis, 1st Edition. WileyInterscience.

Jansen, D. W., de Vries, C. G., 1991. On the Frequency of Large Stock Returns: Putting Booms and Busts Into Perspective. Review of Economics and Statistics 73, 18-24.

Keribin, C., February 2000. Consistent estimation of the order of mixture models. Sankhyā: The Indian Journal of Statistics, Series A 62 (1), 49-66.

Kiefer, J., Wolfowitz, J., 1956. Consistency of the Maximum Likelihood Estimator in the Presence of Infinitely Many Incidental Parameters. The Annals of Mathematical Statistics 27 (4), 887-906.

Kiefer, N. M., 1978. Discrete Parameter Variation: Efficient Estimation of a Switching Regression Model. Econometrica 46 (2), 427-434.

Kim, Y. S., Rachev, S. T., Bianchi, M. L., Fabozzi, F. J., 2008. Financial Market Models with Lévy Processes and Time-Varying Volatility. Journal of Banking \& Finance 32 (7), 1363-1378.

Kim, Y. S., Rachev, S. T., Bianchi, M. L., Fabozzi, F. J., March 2009. Computing VaR and AVaR In Infinitely Divisible Distributions. Yale ICF Working Paper 09-07, Yale School of Management.

Kim, Y. S., Rachev, S. T., Bianchi, M. L., Fabozzi, F. J., 2010. Tempered Stable and Tempered Infinitely Divisible GARCH Models. Journal of Banking \& Finance 34 (9), 2096-2109.

Kirchler, M., Huber, J., 2007. Fat Tails and Volatility Clustering in Experimental Asset Markets. Journal of Economic Dynamics \& Control 31, 1844-1874.

Knight, J., Satchell, S., 2001. Return Distributions in Finance. Butterworth-Heinemann, Oxford.

Kon, S. J., 1984. Models of Stock Returns: A Comparison. The Journal of Finance 39 (1), 147-165.

Kratz, M., Resnick, S. I., 1996. The QQ-Estimator and Heavy Tails. Communications in Statistics - Stochastic Models 12 (4), 699-724.

Kuester, K., Mittnik, S., Paolella, M. S., 2006. Value-at-Risk Prediction: A Comparison of Alternative Strategies. Journal of Financial Econometrics 4 (1), 53-89.

Liu, J.-C., 2007. Stationarity for a Markov-switching Box-Cox Transformed Threshold GARCH Process. Statistics and Probability Letters 77, 1428-1438.

Lombardi, M. J., Veredas, D., 2009. Indirect estimation of elliptical stable distributions. Computational Statistics \& Data Analysis 53 (6), 2309-2324.

Malevergne, Y., Pisarenko, V., Sornette, D., 2005. Empirical Distributions of Stock Returns: Between the Stretched Exponential and the Power Law? Quantitative Finance 5, 379-401.

Mandelbrot, B., 1963. The Variation of Certain Speculative Prices. Journal of Business 36, 394-419.

McCulloch, J. H., 1985. Interest-Risk Sensitive Deposit Insurance Premia: Stable ACH Estimates. Journal of Banking and Finance 9, 137-156.

McCulloch, J. H., 1997. Measuring Tail Thickness in Order to Estimate the Stable Index $\alpha$ : A Critique. Journal of Business and Economic Statistics 15, 74-81.

Mittnik, S., Paolella, M. S., 2003. Prediction of Financial Downside Risk with Heavy Tailed Conditional Distributions. In: Rachev, S. T. (Ed.), Handbook of Heavy Tailed Distributions in Finance. Elsevier Science, Amsterdam.

Mittnik, S., Paolella, M. S., Rachev, S. T., 1998. A Tail Estimator for the Index of the Stable Paretian Distribution. Communications in Statistics-Theory and Methods 27 (5), 1239-1262.

Mittnik, S., Paolella, M. S., Rachev, S. T., 2002. Stationarity of Stable Power-GARCH Processes. Journal of Econometrics 106, 97-107.

Paolella, M. S., 2001. Testing the Stable Paretian Assumption. Mathematical and Computer Modelling 34, 1095-1112.

Paolella, M. S., 2007. Intermediate Probability: A Computational Approach. John Wiley \& Sons, Chichester.

Paolella, M. S., Steude, S. C., 2008. Risk Prediction: A DWARF-like Approach. Journal of Risk Model Validation 2 (1), 25-43.

Rombouts, J. V. K., Bouaddi, M., 2009. Mixed Exponential Power Asymmetric Conditional Heteroskedas- 
ticity. Studies in Nonlinear Dynamics \& Econometrics 13 (3), Article 3.

Samanidou, E., Zschischang, E., Stauffer, D., 2007. Agent-Based Models of Financial Markets. Reports on Progress in Physics 70, 409-450.

Samorodnitsky, G., Taqqu, M. S., 1994. Stable Non-Gaussian Random Processes: Stochastic Models with Infinite Variance. Chapman \& Hall, London.

Stoyanov, S., Samorodnitsky, G., Rachev, S., Ortobelli, S., 2006. Computing the Portfolio Conditional Value-at-Risk in the alpha-stable Case. Probability and Mathematical Statistics 26, 1-22.

Sy, W., 2006. On the Coherence of VaR Risk Measure for Levy Stable Distributions. Working papers, Australian Prudential Regulation Authority.

Tanaka, K., 2009. Strong Consistency of the Maximum Likelihood Estimator for Finite Mixtures of LocationScale Distributions When Penalty is Imposed on the Ratios of the Scale Parameters. Scandinavian Journal of Statistics 36, 171-184.

Vigfusson, R., 1997. Switching between Chartists and Fundamentalists: A Markov Regime-Switching Approach. International Journal of Finance and Economics 2, 291-305.

Vlaar, P. J. G., Palm, F. C., 1993. The Message in Weekly Exchange Rates in the European Monetary System: Mean Reversion, Conditional Heteroscedasticity, and Jumps. Journal of Business and Economic Statistics 11 (3), 351-360.

Weron, R., 2001. Levy-stable Distributions Revisited: Tail Index $>2$ does not exclude the Levy-stable Regime. International Journal of Modern Physics C 12, 209-223. 
MixStable $(3,3)$

\begin{tabular}{|c|c|c|c|c|c|c|c|c|c|c|c|c|c|c|c|c|}
\hline$\gamma_{0,1}$ & $\gamma_{0,2}$ & $\gamma_{0,3}$ & $\gamma_{1,1}$ & $\gamma_{2,1}$ & $\gamma_{3,1}$ & $\Psi_{1}$ & $\Psi_{2}$ & $\Psi_{3}$ & $\omega_{1}$ & $\omega_{2}$ & & $\mu_{1}$ & $\mu_{2}$ & $\mu_{3}$ & $\alpha$ & $\beta$ \\
\hline 1.012 & $4 e-13$ & 0.01 & 0.807 & $7 e-3$ & 0.089 & 0.648 & 0.984 & 0.916 & 0.019 & 0.346 & 0.635 & -1.277 & 0.118 & -0.025 & 2 & 0 \\
\hline
\end{tabular}
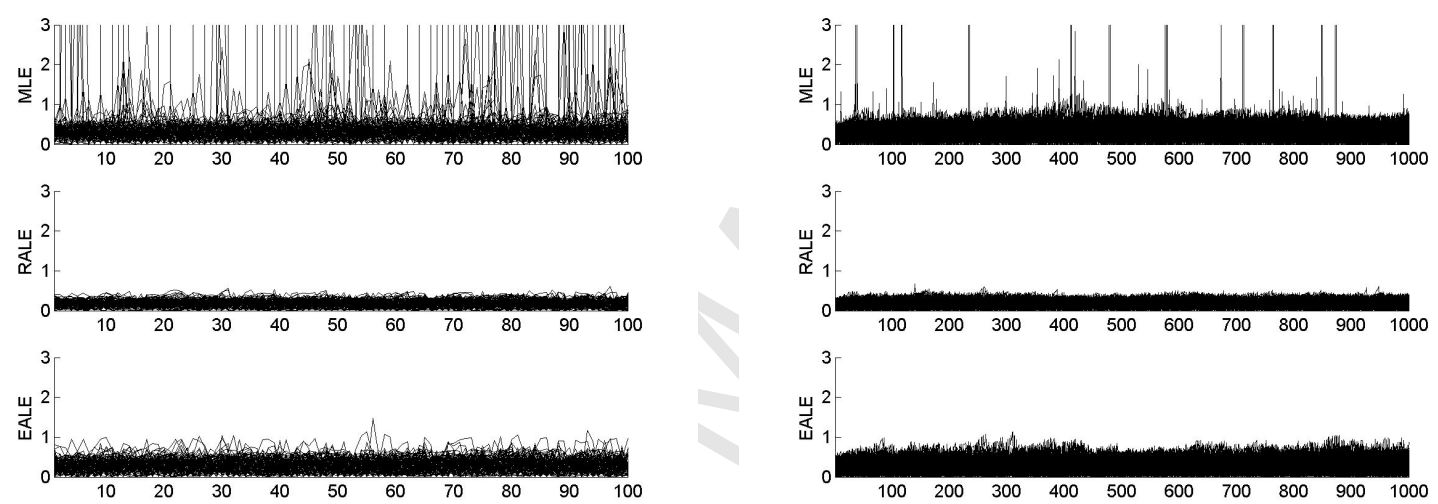

sample size 100

sample size 1000

Figure 1: Comparison of MLE, RALE and EALE in presence of local mixture degeneracy. Each panel shows 100 overlaid (maximized) likelihood series (not the augmented likelihood), from which the usual likelihood function is computed, obtained from estimates based on simulated data. Spikes depict either mixture degeneracy (singularities) or almost degenerated local optima. Results are essentially identical for all mixture GARCH models under study. The data generating process roughly corresponds to estimates found in our empirical analysis of DJIA return data. 


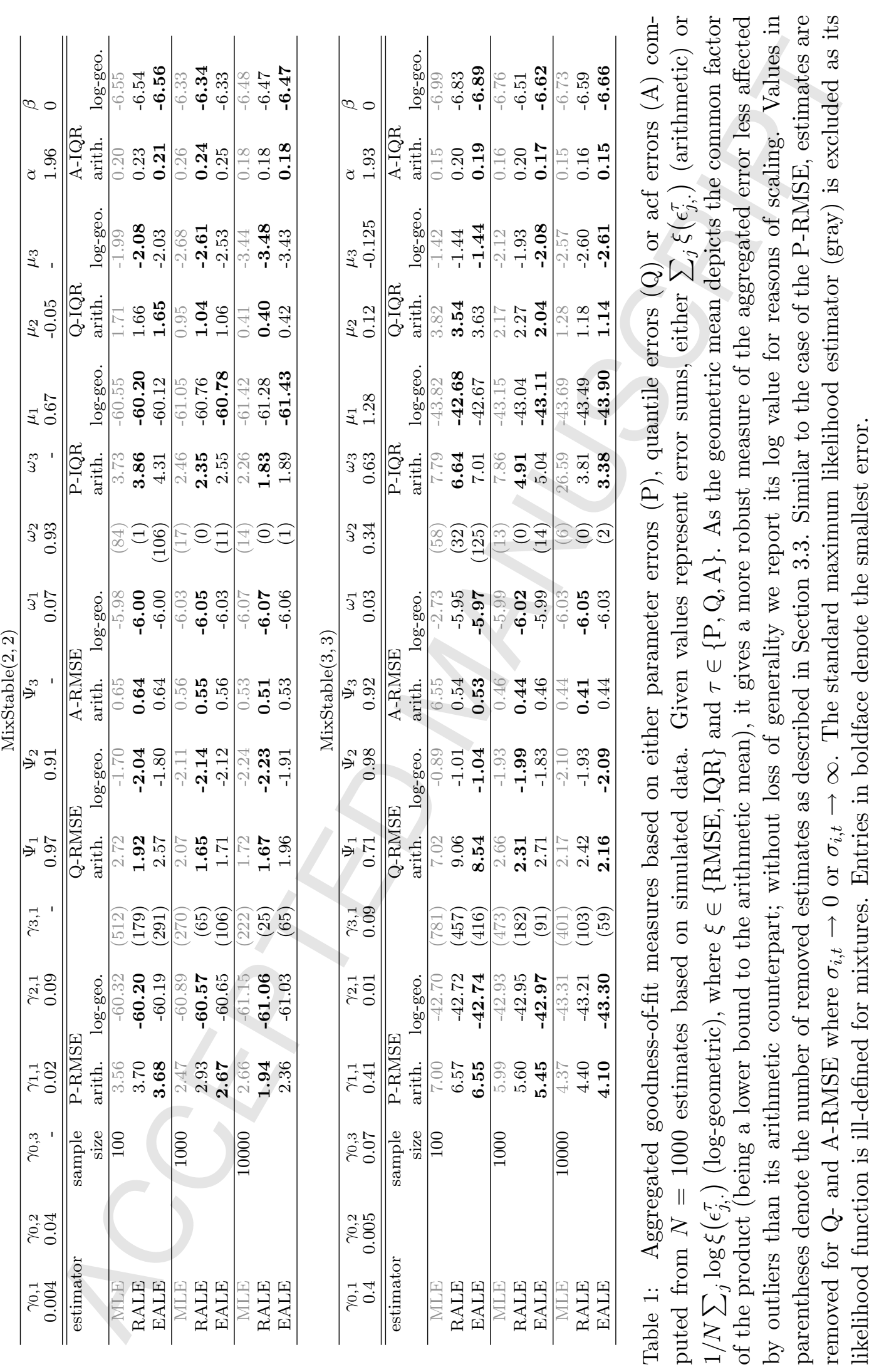




\begin{tabular}{|c|c|c|c|c|c|c|c|c|}
\hline & $\begin{array}{c}\text { MixNormal } \\
(2,2)\end{array}$ & $(3,2)$ & $(3,3)$ & $(4,4)$ & $\begin{array}{r}\text { MixGED } \\
(2,2)\end{array}$ & $(3,2)$ & $(3,3)$ & $(4,4)$ \\
\hline DAX & 16310.03 & 16288.08 & 6305.12 & 6337.01 & 16309.17 & 16296.28 & 16313.32 & 16345.54 \\
\hline $\mathrm{S} \& \mathrm{P}$ & 500.95 & 13470.42 & 3483.12 & 509.51 & 13463.34 & 13475.66 & 3490.43 & 3518.04 \\
\hline DJIA & 13388.74 & 13376.78 & 13389.46 & 13418.75 & 13366.56 & 13377.48 & 13392.69 & 13427.38 \\
\hline NIKKEI & 17020.03 & 17026.97 & 17040.97 & 17071.84 & 17018.64 & 17032.31 & 17047.74 & 17080.34 \\
\hline$¥ / €$ & 5332.19 & 5351.45 & 5361.39 & 5396.60 & 5340.00 & 5359.22 & 5369.22 & 5404.44 \\
\hline$\$ / €$ & 4972.80 & 4985.69 & 5000.25 & 5035.42 & 4963.06 & 4985.51 & 5000.62 & 5039.79 \\
\hline NAS & 16395.36 & 16397.37 & 16402.99 & 16430.04 & 16393.98 & 16404.58 & 16411.53 & 16438.58 \\
\hline \multirow[t]{2}{*}{ free param. } & 8 & 11 & 13 & 18 & +8 & 12 & 14 & 19 \\
\hline & $\begin{array}{c}\mathrm{A}^{1} \text { MixStable } \\
(2,2) \\
\end{array}$ & $(3,2)$ & $(3,3)$ & $(4,4)$ & $\begin{array}{c}\mathrm{A}^{2} \text { MixStak } \\
(2,2)\end{array}$ & $(3,2)$ & $(3,3)$ & $(4,4)$ \\
\hline DAX & 16282.68 & 16296.61 & 16312.39 & 16345.30 & 16283.34 & 16300.37 & 16317.15 & 16351.25 \\
\hline S\&P & 13476.56 & 13478.95 & 13491.62 & 13517.74 & 13460.74 & 13477.79 & 3483.34 & 13517.33 \\
\hline DJIA & 13380.83 & 13385.32 & & 13426.46 & 13366.22 & 13383.26 & .68 & 13423.44 \\
\hline NIKKI & 17018.93 & 17035.48 & 17046.67 & 17079.53 & 17026.53 & 17035.61 & 17046.74 & 17074.25 \\
\hline$¥ / €$ & 5339.27 & 5359.29 & 5369.23 & 5404.42 & 5347.54 & 5363.20 & 5378.66 & 5409.64 \\
\hline$\$ / €$ & 4970.42 & 4989.10 & 5001.91 & 5036.64 & 4971.85 & 4985.76 & 4999.43 & 5028.71 \\
\hline NASDAQ & 16396.48 & 16377.25 & 16403.87 & 16431.01 & 16370.55 & 16388.71 & 16392.76 & 16423.96 \\
\hline free param. & 9 & 12 & 14 & 19 & 9 & 12 & 13 & 17 \\
\hline
\end{tabular}

Table 2: BIC values for all multi-component mixture GARCH models using $\delta=1$ and all data sets under study (20 years of equity returns, 10 years of FX returns). Entries in boldface denote the best results per data set. 


\begin{tabular}{|c|c|c|c|c|c|c|c|}
\hline model & DAX & $\mathrm{S} \& \mathrm{P}$ & DJIA & NIKKEI & $¥ / €$ & $\$ / €$ & NASDAQ \\
\hline \multicolumn{8}{|l|}{ Anderson-Darling } \\
\hline MixNormal $(2,2)$ & 0.86 & 1.00 & 1.27 & 0.93 & 1.54 & 1.52 & 1.32 \\
\hline $\operatorname{MixNormal}(3,2)$ & 0.65 & 0.54 & 0.59 & 0.73 & 1.49 & 1.33 & 0.43 \\
\hline $\operatorname{MixNormal}(3,3)$ & 0.55 & 0.53 & 0.54 & 0.60 & 1.41 & 1.51 & 0.42 \\
\hline $\operatorname{MixNormal}(4,4)$ & 0.69 & 0.37 & 0.42 & 0.54 & 1.22 & 1.59 & 0.47 \\
\hline $\operatorname{Mix} \operatorname{GED}(2,2)$ & 0.72 & 0.63 & 0.67 & 0.73 & 1.44 & 1.41 & 0.30 \\
\hline $\operatorname{Mix} \operatorname{GED}(3,2)$ & 0.76 & 0.56 & 0.52 & 0.62 & 1.45 & 1.09 & 0.52 \\
\hline $\operatorname{Mix} \operatorname{GED}(3,3)$ & 0.68 & 0.51 & 0.49 & 0.48 & 1.45 & 1.30 & 0.46 \\
\hline $\operatorname{Mix} \operatorname{GED}(4,4)$ & 0.72 & 0.41 & 0.59 & 0.45 & 1.21 & 1.47 & 0.51 \\
\hline $\mathrm{A}^{1} \operatorname{MixStable}(2,2)$ & 0.73 & 0.49 & 0.55 & 0.92 & 1.36 & 1.20 & 0.35 \\
\hline $\mathrm{A}^{1}$ MixStable $(3,2)$ & 0.70 & 0.45 & 0.43 & 0.65 & 1.48 & 1.01 & 0.31 \\
\hline $\mathrm{A}^{1} \operatorname{MixStable}(3,3)$ & 0.66 & 0.43 & 0.36 & 0.44 & 1.43 & 1.38 & 0.36 \\
\hline $\mathrm{A}^{1} \operatorname{MixStable}(4,4)$ & 0.63 & 0.31 & 0.28 & 0.34 & 1.24 & 1.48 & 0.44 \\
\hline $\mathrm{A}^{2} \operatorname{MixStable}(2,2)$ & 1.12 & 1.05 & 0.67 & 0.59 & 0.84 & 1.88 & 1.61 \\
\hline $\mathrm{A}^{2} \operatorname{MixStable}(3,2)$ & 1.16 & 1.02 & 0.58 & 0.58 & 0.85 & 1.71 & 1.59 \\
\hline $\mathrm{A}^{2}$ MixStable $(3,3)$ & 1.22 & 0.95 & 0.56 & 0.58 & 0.85 & 1.68 & 1.59 \\
\hline $\mathrm{A}^{2} \operatorname{MixStable}(4,4)$ & 1.23 & 0.99 & 0.56 & 0.59 & 0.86 & 1.70 & 1.63 \\
\hline \multicolumn{8}{|l|}{ Cramér-von Mises } \\
\hline MixNormal $(2,2)$ & 0.12 & 0.12 & 0.22 & 0.16 & 0.30 & 0.30 & 0.23 \\
\hline $\operatorname{MixNormal}(3,2)$ & 0.06 & 0.07 & 0.07 & 0.12 & 0.27 & 0.25 & 0.04 \\
\hline $\operatorname{MixNormal}(3,3)$ & 0.06 & 0.08 & 0.06 & 0.08 & 0.25 & 0.28 & 0.04 \\
\hline $\operatorname{MixNormal}(4,4)$ & 0.07 & 0.06 & 0.05 & 0.08 & 0.22 & 0.29 & 0.04 \\
\hline $\operatorname{Mix} \operatorname{GED}(2,2)$ & 0.09 & 0.08 & 0.09 & 0.10 & 0.27 & 0.26 & 0.03 \\
\hline $\operatorname{Mix} \operatorname{GED}(3,2)$ & 0.08 & 0.07 & 0.06 & 0.09 & 0.26 & 0.20 & 0.05 \\
\hline $\operatorname{Mix} \operatorname{GED}(3,3)$ & 0.07 & 0.07 & 0.06 & 0.06 & 0.26 & 0.24 & 0.04 \\
\hline $\operatorname{Mix} \operatorname{GED}(4,4)$ & 0.07 & 0.05 & 0.07 & 0.06 & 0.22 & 0.27 & 0.05 \\
\hline $\mathrm{A}^{1} \operatorname{MixStable}(2,2)$ & 0.09 & 0.07 & 0.08 & 0.15 & 0.25 & 0.23 & 0.04 \\
\hline $\mathrm{A}^{1} \operatorname{MixStable}(3,2)$ & 0.08 & 0.05 & 0.05 & 0.10 & 0.27 & 0.19 & 0.03 \\
\hline $\mathrm{A}^{1} \operatorname{MixStable}(3,3)$ & 0.06 & 0.06 & 0.04 & 0.05 & 0.26 & 0.24 & 0.04 \\
\hline $\mathrm{A}^{1}$ MixStable $(4,4)$ & 0.06 & 0.05 & 0.03 & 0.04 & 0.22 & 0.26 & 0.04 \\
\hline $\mathrm{A}^{2} \operatorname{MixStable}(2,2)$ & 0.14 & 0.15 & 0.10 & 0.07 & 0.15 & $0.41 *$ & 0.27 \\
\hline $\mathrm{A}^{2}$ MixStable $(3,2)$ & 0.14 & 0.14 & 0.08 & 0.06 & 0.16 & $0.37 *$ & 0.26 \\
\hline $\mathrm{A}^{2}$ MixStable $(3,3)$ & 0.15 & 0.13 & 0.07 & 0.06 & 0.16 & $0.36^{*}$ & 0.27 \\
\hline $\mathrm{A}^{2} \operatorname{MixStable}(4,4)$ & 0.15 & 0.14 & 0.07 & 0.06 & 0.16 & $0.37^{*}$ & 0.27 \\
\hline \multicolumn{8}{|c|}{ Kolmogorov-Smirnov (test statistics are scaled up by factor 100) } \\
\hline MixNormal $(2,2)$ & 1.48 & 1.44 & 1.97 & 1.73 & 3.23 & $3.76^{*}$ & 2.41 \\
\hline $\operatorname{MixNormal}(3,2)$ & 1.28 & 1.64 & 1.36 & 1.47 & 3.39 & 3.47 & 1.07 \\
\hline $\operatorname{MixNormal}(3,3)$ & 1.19 & 1.57 & 1.25 & 1.25 & 3.40 & 3.58 & $0.96^{*}$ \\
\hline $\operatorname{MixNormal}(4,4)$ & 1.38 & 1.32 & 1.17 & 1.34 & 3.12 & 3.71 & 1.19 \\
\hline $\operatorname{MixGED}(2,2)$ & 1.33 & 1.62 & 1.33 & 1.44 & 3.13 & 3.63 & 1.09 \\
\hline $\operatorname{Mix} \operatorname{GED}(3,2)$ & 1.36 & 1.47 & 1.21 & 1.26 & 3.39 & 2.77 & 1.05 \\
\hline $\operatorname{Mix} \operatorname{GED}(3,3)$ & 1.33 & 1.50 & 1.36 & 1.15 & 3.40 & 3.33 & $0.97^{*}$ \\
\hline $\operatorname{Mix} \operatorname{GED}(4,4)$ & 1.42 & 1.06 & 1.34 & 1.03 & 3.19 & 3.37 & 1.06 \\
\hline $\mathrm{A}^{1} \operatorname{MixStable}(2,2)$ & 1.29 & 1.25 & 1.31 & 1.73 & 3.11 & 3.14 & 1.11 \\
\hline $\mathrm{A}^{1} \operatorname{MixStable}(3,2)$ & 1.27 & 1.54 & 1.21 & 1.59 & 3.32 & 2.79 & $0.86^{* *}$ \\
\hline $\mathrm{A}^{1} \operatorname{MixStable}(3,3)$ & 1.33 & 1.48 & 1.21 & 1.10 & 3.40 & 3.33 & 1.04 \\
\hline $\mathrm{A}^{1} \operatorname{MixStable}(4,4)$ & 1.28 & 1.11 & 1.02 & $0.90^{* *}$ & 3.25 & 3.35 & 1.14 \\
\hline $\mathrm{A}^{2} \operatorname{MixStable}(2,2)$ & 2.17 & 1.63 & 1.74 & 1.54 & 2.86 & $4.27^{* *}$ & 2.18 \\
\hline $\mathrm{A}^{2} \operatorname{MixStable}(3,2)$ & 2.21 & 1.79 & 1.30 & 1.41 & 2.87 & $3.98 *$ & 2.03 \\
\hline $\mathrm{A}^{2} \operatorname{MixStable}(3,3)$ & 2.21 & 1.67 & 1.26 & 1.46 & 2.89 & $3.88^{*}$ & 2.16 \\
\hline $\mathrm{A}^{2} \operatorname{MixStable}(4,4)$ & 2.20 & 1.77 & 1.32 & 1.51 & 2.89 & $3.88^{*}$ & 2.16 \\
\hline
\end{tabular}

Table 3: Anderson-Darling, Cramér-von Mises and Kolmogorov-Smirnov test statistics for all models (with $\delta=1$ ) and data sets under study. Entries in boldface denote the best outcomes. ***, **, and $*$ denote significance at the $1 \%, 5 \%$, and $10 \%$ levels, respectively. All values are based on evaluating the one-step-ahead out-of-sample distribution forecasts at the observed return data, estimated throughout a rolling window of 1,000 trading days (updated every 20 days), covering 10 years of equity returns (July 7, 1999 to July 7, 2009) and 5 years (July 7, 2004 to July 7, 2009) of FX returns. 


\begin{tabular}{|c|c|c|c|c|c|c|c|}
\hline model & DAX & $\mathrm{S} \& \mathrm{P}$ & DJIA & NIKKEI & $¥ / €$ & $\$ / €$ & NASDAQ \\
\hline \multicolumn{8}{|c|}{ Ljung-Box $(m=20$ lags $)$} \\
\hline MixNormal $(2,2)$ & 26.44 & $32.97 *$ & 30.66 & 16.95 & 17.46 & 18.57 & 31.05 \\
\hline $\operatorname{MixNormal}(3,2)$ & 26.74 & $33.15^{*}$ & 30.46 & 17.01 & 17.59 & 18.55 & $31.62 *$ \\
\hline $\operatorname{MixNormal}(3,3)$ & 26.63 & $33.26^{*}$ & 30.68 & 17.49 & 17.65 & 18.50 & 30.92 \\
\hline $\operatorname{MixNormal}(4,4)$ & 26.25 & $33.77^{*}$ & 30.78 & 17.95 & 17.99 & 18.03 & $31.86^{*}$ \\
\hline $\operatorname{Mix} \operatorname{GED}(2,2)$ & 26.62 & $33.61^{*}$ & 31.12 & 16.94 & 17.46 & 17.55 & 30.92 \\
\hline $\operatorname{Mix} \operatorname{GED}(3,2)$ & 26.73 & $33.15^{*}$ & 30.69 & 17.12 & 17.78 & 17.95 & $31.83^{*}$ \\
\hline $\operatorname{Mix} \operatorname{GED}(3,3)$ & 26.90 & $33.41^{*}$ & 31.33 & 17.96 & 17.64 & 17.96 & 31.37 \\
\hline $\operatorname{Mix} \operatorname{GED}(4,4)$ & 26.51 & $33.61^{*}$ & 30.62 & 18.10 & 17.82 & 18.20 & $31.46^{*}$ \\
\hline $\mathrm{A}^{1} \operatorname{MixStable}(2,2)$ & 26.46 & $32.87^{*}$ & 29.83 & 16.96 & 17.34 & 18.49 & 30.49 \\
\hline $\mathrm{A}^{1} \operatorname{MixStable}(3,2)$ & 26.29 & $33.35^{*}$ & 30.63 & 19.64 & 17.67 & 19.17 & $31.50 *$ \\
\hline $\mathrm{A}^{1} \operatorname{MixStable}(3,3)$ & 26.33 & $33.55^{*}$ & 30.72 & 17.59 & 17.77 & 17.94 & 30.78 \\
\hline $\mathrm{A}^{1} \operatorname{MixStable}(4,4)$ & 26.44 & $33.94^{*}$ & 31.25 & 18.24 & 17.67 & 18.29 & $31.45^{*}$ \\
\hline $\mathrm{A}^{2}$ MixStable $(2,2)$ & 27.21 & $33.45^{*}$ & 30.00 & 17.83 & 16.17 & 17.66 & 31.30 \\
\hline $\mathrm{A}^{2}$ MixStable $(3,2)$ & 26.95 & $33.12^{*}$ & 29.86 & 17.92 & 16.14 & 17.74 & $31.58^{*}$ \\
\hline $\mathrm{A}^{2} \operatorname{MixStable}(3,3)$ & 27.00 & $33.14^{*}$ & 29.99 & 17.89 & 16.22 & 17.94 & $31.55^{*}$ \\
\hline $\mathrm{A}^{2} \operatorname{MixStable}(4,4)$ & 27.04 & $33.14^{*}$ & 29.85 & 17.91 & 16.21 & 17.86 & $31.42^{*}$ \\
\hline \multicolumn{8}{|l|}{ Jarque-Bera } \\
\hline $\operatorname{MixNormal}(2,2)$ & $28.31^{* * *}$ & $51.49^{* * *}$ & $18.88^{* * *}$ & 2.11 & $9.73^{* *}$ & $9.71^{* *}$ & $80.46^{* * *}$ \\
\hline $\operatorname{MixNormal}(3,2)$ & $17.25^{* * *}$ & 3.01 & 5.69 & 3.19 & $14.94^{* * *}$ & $17.53^{* * *}$ & $7.01^{*}$ \\
\hline $\operatorname{MixNormal}(3,3)$ & $19.61^{* * *}$ & $198.32^{* * *}$ & $52.25 * * *$ & 4.95 & $22.28^{* * *}$ & $16.10^{* * *}$ & $65.26 * * *$ \\
\hline $\operatorname{MixNormal}(4,4)$ & 4.61 & $70.44^{* * *}$ & 5.57 & 5.42 & $13.52^{* * *}$ & $20.68^{* * *}$ & $56.53 * * *$ \\
\hline $\operatorname{Mix} \operatorname{GED}(2,2)$ & 2.24 & $9.63^{* *}$ & $9.63^{* *}$ & 3.14 & $9.82^{* *}$ & $6.64^{*}$ & $19.23^{* * *}$ \\
\hline $\operatorname{Mix} \operatorname{GED}(3,2)$ & $15.18^{* * *}$ & $9.35^{* *}$ & $9.94^{* *}$ & 3.43 & $14.12^{* * *}$ & 3.79 & $7.23^{*}$ \\
\hline $\operatorname{Mix} \operatorname{GED}(3,3)$ & $17.54^{* * *}$ & $12.13^{* * *}$ & 4.45 & 4.22 & $19.36^{* * *}$ & 5.50 & $18.01^{* * *}$ \\
\hline $\operatorname{Mix} \operatorname{GED}(4,4)$ & 4.97 & $9.10^{* *}$ & $9.06^{* *}$ & 4.69 & $15.94^{* * *}$ & $6.67^{*}$ & $16.18^{* * *}$ \\
\hline $\mathrm{A}^{1} \operatorname{MixStable}(2,2)$ & 2.06 & 1.28 & $8.40^{* *}$ & 2.23 & $10.47^{* *}$ & 5.49 & 0.80 \\
\hline $\mathrm{A}^{1} \operatorname{MixStable}(3,2)$ & 2.04 & 1.63 & 2.72 & 3.31 & $14.52^{* * *}$ & 4.14 & 1.28 \\
\hline $\mathrm{A}^{1} \operatorname{MixStable}(3,3)$ & 1.31 & 2.47 & 2.22 & 5.74 & $18.98 * * *$ & 4.88 & 1.71 \\
\hline $\mathrm{A}^{1} \operatorname{MixStable}(4,4)$ & 1.17 & 0.79 & 1.45 & 3.51 & $10.79^{* * *}$ & $7.91^{* *}$ & 2.23 \\
\hline $\mathrm{A}^{2}$ MixStable $(2,2)$ & 0.28 & $0.06^{*}$ & 0.54 & 2.75 & 3.70 & $8.56^{* *}$ & 0.38 \\
\hline $\mathrm{A}^{2}$ MixStable $(3,2)$ & 0.32 & $0.05 * *$ & 0.39 & 2.83 & 3.41 & $6.10^{*}$ & 1.77 \\
\hline $\mathrm{A}^{2}$ MixStable $(3,3)$ & 0.67 & 0.23 & 0.42 & 3.31 & 3.78 & 3.13 & $0.03 * *$ \\
\hline $\mathrm{A}^{2}$ MixStable $(4,4)$ & 0.69 & 0.25 & 0.51 & 3.11 & 3.41 & 3.25 & 0.17 \\
\hline \multicolumn{8}{|c|}{ Shapiro-Wilk (test statistic $\nu$ is transformed by $1000(1-\nu)$ ) } \\
\hline $\operatorname{MixNormal}(2,2)$ & $3.16^{* * *}$ & $3.87 * * *$ & $2.65^{* * *}$ & 1.23 & $3.11^{* *}$ & 2.01 & $6.04^{* * *}$ \\
\hline $\operatorname{MixNormal}(3,2)$ & $2.32^{* * *}$ & 0.94 & $1.68^{* *}$ & $1.32^{*}$ & $4.00 * * *$ & $2.91^{* *}$ & $1.63^{* *}$ \\
\hline $\operatorname{MixNormal}(3,3)$ & $2.40 * * *$ & $6.94 * * *$ & $4.53^{* * *}$ & $1.65^{* *}$ & $4.72^{* * *}$ & $2.69^{*}$ & $5.37 * * *$ \\
\hline $\operatorname{MixNormal}(4,4)$ & 1.16 & $4.17 * * *$ & $1.44^{* *}$ & $1.60^{* *}$ & $3.37^{* *}$ & $3.13^{* *}$ & $5.43 * * *$ \\
\hline $\operatorname{MixGED}(2,2)$ & 0.92 & $1.78^{* *}$ & $2.23 * * *$ & 1.25 & $3.10^{* *}$ & 1.74 & $3.05^{* * *}$ \\
\hline $\operatorname{Mix} \operatorname{GED}(3,2)$ & $2.27 * * *$ & $1.72^{* *}$ & $2.30 * * *$ & $1.27^{*}$ & $3.87^{* * *}$ & 1.01 & $2.22^{* * *}$ \\
\hline $\operatorname{Mix} \operatorname{GED}(3,3)$ & $2.25 * * *$ & $1.93^{* * *}$ & $1.30^{*}$ & $1.52^{* *}$ & $4.49^{* * *}$ & 1.49 & $3.68 * * *$ \\
\hline $\operatorname{Mix} \operatorname{GED}(4,4)$ & 1.19 & $1.50 * *$ & $2.24^{* * *}$ & $1.50^{* *}$ & $3.87^{* * *}$ & 1.55 & $3.62^{* * *}$ \\
\hline $\mathrm{A}^{1} \operatorname{MixStable}(2,2)$ & 0.67 & 0.58 & $1.78^{* *}$ & $1.31^{*}$ & $3.25^{* *}$ & 1.32 & 0.91 \\
\hline $\mathrm{A}^{1} \operatorname{MixStable}(3,2)$ & 0.57 & 0.55 & 1.00 & $1.27^{*}$ & $3.93^{* * *}$ & 1.17 & 0.82 \\
\hline $\mathrm{A}^{1}$ MixStable $(3,3)$ & 0.57 & 0.59 & 0.90 & $1.56^{* *}$ & $4.43^{* * *}$ & 1.35 & 1.23 \\
\hline $\mathrm{A}^{1} \operatorname{MixStable}(4,4)$ & 0.56 & 0.41 & 0.81 & $1.34^{*}$ & $3.12^{* *}$ & 1.86 & $1.43^{* *}$ \\
\hline $\mathrm{A}^{2}$ MixStable $(2,2)$ & $1.51^{* *}$ & 1.16 & 0.75 & $1.26^{*}$ & 2.06 & 2.19 & $1.76^{* *}$ \\
\hline $\mathrm{A}^{2} \operatorname{MixStable}(3,2)$ & $1.55^{* *}$ & 1.15 & 0.74 & $1.33^{*}$ & 2.14 & 1.85 & $1.73^{* *}$ \\
\hline $\mathrm{A}^{2}$ MixStable $(3,3)$ & $1.43^{* *}$ & 1.15 & 0.77 & $1.27^{*}$ & 2.11 & 1.34 & $1.75^{* *}$ \\
\hline $\mathrm{A}^{2} \operatorname{MixStable}(4,4)$ & $1.44^{* *}$ & 1.08 & 0.76 & $1.28^{*}$ & 2.14 & 1.36 & $1.85^{* * *}$ \\
\hline
\end{tabular}

Table 4: Ljung-Box, Jarque-Bera and Shapiro-Wilk test statistics for all models (with $\delta=1$ ) and data sets under study. Entries in boldface denote the best outcomes. ***, **, and * denote significance at the $1 \%, 5 \%$, and $10 \%$ levels, respectively. Results are based on the same out-ofsample exercise as in Table 3. 


\begin{tabular}{|c|c|c|c|c|c|c|c|}
\hline model & DAX & $\mathrm{S} \& \mathrm{P}$ & DJIA & NIKKEI & $¥ / €$ & $\$ / €$ & NASDAQ \\
\hline \multicolumn{8}{|l|}{$\overline{1 \% \mathrm{VaR}}$} \\
\hline MixNormal $(2,2)$ & 1.01 & 1.11 & 1.21 & 0.87 & $1.60^{* *}$ & 1.15 & 0.96 \\
\hline $\operatorname{MixNormal}(3,2)$ & 0.96 & 1.08 & 1.06 & 0.87 & $1.68^{* *}$ & 1.11 & 0.60 \\
\hline $\operatorname{MixNormal}(3,3)$ & 0.84 & 1.09 & 1.13 & 0.84 & $1.63^{* *}$ & 1.20 & 0.57 \\
\hline $\operatorname{MixNormal}(4,4)$ & 0.92 & 1.12 & 1.03 & 0.89 & $1.62^{* *}$ & 1.19 & 0.61 \\
\hline $\operatorname{MixGED}(2,2)$ & 1.00 & 1.11 & 1.11 & 0.90 & $1.75^{* *}$ & 1.40 & 0.81 \\
\hline $\operatorname{Mix} \operatorname{GED}(3,2)$ & 0.96 & 1.18 & 1.07 & 0.87 & $1.67^{* *}$ & 1.11 & 0.63 \\
\hline $\operatorname{Mix} \operatorname{GED}(3,3)$ & 0.88 & 1.10 & 1.03 & 0.80 & $1.76^{* *}$ & 1.32 & 0.59 \\
\hline $\operatorname{Mix} \operatorname{GED}(4,4)$ & 0.88 & 1.10 & 1.03 & 0.85 & $1.72^{* *}$ & 1.20 & 0.64 \\
\hline $\mathrm{A}^{1} \operatorname{MixStable}(2,2)$ & 1.11 & 1.13 & 1.11 & 0.87 & $1.75^{* *}$ & 1.16 & 0.68 \\
\hline $\mathrm{A}^{1} \operatorname{MixStable}(3,2)$ & 1.09 & 1.17 & 1.03 & 0.88 & $1.60^{* *}$ & 1.13 & 0.62 \\
\hline $\mathrm{A}^{1} \operatorname{MixStable}(3,3)$ & 1.02 & 1.14 & 1.17 & 0.85 & $1.63^{* *}$ & 1.22 & 0.70 \\
\hline $\mathrm{A}^{1} \operatorname{MixStable}(4,4)$ & 0.88 & 1.06 & 1.02 & 0.92 & $1.63^{* *}$ & 1.20 & 0.67 \\
\hline $\mathrm{A}^{2} \operatorname{MixStable}(2,2)$ & 0.77 & 0.98 & 1.05 & 0.90 & 1.46 & 1.13 & 0.71 \\
\hline $\mathrm{A}^{2}$ MixStable $(3,2)$ & 0.77 & 0.98 & 1.07 & 0.91 & 1.40 & 1.09 & 0.68 \\
\hline $\mathrm{A}^{2}$ MixStable $(3,3)$ & 0.77 & 0.98 & 1.05 & 0.98 & 1.42 & 1.08 & 0.64 \\
\hline $\mathrm{A}^{2} \operatorname{MixStable}(4,4)$ & 0.78 & 0.98 & 1.04 & 0.93 & 1.46 & 1.09 & 0.66 \\
\hline \multicolumn{8}{|l|}{$\overline{5 \% \mathrm{VaR}}$} \\
\hline $\operatorname{MixNormal}(2,2)$ & 5.42 & 5.48 & 5.46 & $5.83^{* *}$ & 5.02 & 4.28 & 5.49 \\
\hline $\operatorname{MixNormal}(3,2)$ & 5.38 & 5.35 & 5.22 & $5.70^{*}$ & 5.15 & 3.96 & 4.81 \\
\hline $\operatorname{MixNormal}(3,3)$ & 5.23 & 5.20 & 5.22 & 5.58 & 5.17 & 4.01 & 4.60 \\
\hline $\operatorname{MixNormal}(4,4)$ & 5.33 & 5.04 & 5.19 & 5.56 & 5.18 & 3.96 & 4.45 \\
\hline $\operatorname{Mix} \operatorname{GED}(2,2)$ & 5.30 & 5.20 & 5.45 & $5.90^{* *}$ & 5.02 & 4.51 & 5.09 \\
\hline $\operatorname{Mix} \operatorname{GED}(3,2)$ & 5.22 & 5.34 & 5.30 & $5.77^{* *}$ & 5.33 & 4.15 & 4.61 \\
\hline $\operatorname{Mix} \operatorname{GED}(3,3)$ & 5.26 & 5.13 & 5.35 & $5.80^{* *}$ & 5.23 & 4.44 & 4.58 \\
\hline $\operatorname{Mix} \operatorname{GED}(4,4)$ & 5.33 & 5.22 & 5.30 & 5.52 & 5.20 & 4.24 & 4.49 \\
\hline $\mathrm{A}^{1} \operatorname{MixStable}(2,2)$ & 5.46 & 5.33 & 5.15 & $5.86^{* *}$ & 5.10 & 4.06 & 5.04 \\
\hline $\mathrm{A}^{1}$ MixStable $(3,2)$ & 5.27 & 5.21 & 5.05 & 5.49 & 5.15 & 3.95 & 4.83 \\
\hline $\mathrm{A}^{1}$ MixStable $(3,3)$ & 5.31 & 5.14 & 5.05 & $5.63^{*}$ & 5.15 & 4.20 & 4.37 \\
\hline $\mathrm{A}^{1} \operatorname{MixStable}(4,4)$ & 5.29 & 4.94 & 5.03 & 5.48 & 5.20 & 4.19 & 4.26 \\
\hline $\mathrm{A}^{2}$ MixStable $(2,2)$ & 5.47 & 5.47 & 5.36 & $5.67^{*}$ & 5.55 & 4.61 & $5.61^{*}$ \\
\hline $\mathrm{A}^{2}$ MixStable $(3,2)$ & 5.46 & 5.46 & 5.32 & $5.67 *$ & 5.55 & 4.61 & 5.40 \\
\hline $\mathrm{A}^{2} \operatorname{MixStable}(3,3)$ & 5.51 & 5.40 & 5.28 & $5.60^{*}$ & 5.65 & 4.54 & 5.37 \\
\hline $\mathrm{A}^{2} \operatorname{MixStable}(4,4)$ & 5.51 & 5.43 & 5.26 & $5.64^{*}$ & 5.64 & 4.53 & 5.52 \\
\hline
\end{tabular}

Table 5: Predicted VaR coverage percentages (point estimates) at the $1 \%$ and $5 \%$ level for all models under study (with $\delta=1$ ). Entries in boldface denote the best (closest to the true value) estimate. $* * *, * *$, and $*$ denote significance at the $1 \%, 5 \%$, and $10 \%$ levels, respectively. Results are based on the same out-of-sample exercise as in Table 3. 


\begin{tabular}{|c|c|c|c|c|c|c|c|}
\hline model & DAX & $\mathrm{S} \& \mathrm{P}$ & DJIA & NIKKEI & $¥ / €$ & $\$ / €$ & NASDAQ \\
\hline \multicolumn{8}{|l|}{$1 \% \mathrm{VaR}$} \\
\hline $\operatorname{MixNormal}(2,2)$ & 0.10 & 0.22 & 0.12 & 0.11 & 0.32 & 0.10 & 0.06 \\
\hline $\operatorname{MixNormal}(3,2)$ & 0.09 & 0.15 & 0.13 & 0.08 & 0.34 & 0.12 & 0.29 \\
\hline $\operatorname{MixNormal}(3,3)$ & 0.08 & 0.10 & 0.10 & 0.10 & 0.33 & 0.16 & 0.31 \\
\hline $\operatorname{MixNormal}(4,4)$ & 0.05 & 0.12 & 0.08 & 0.08 & 0.29 & 0.17 & 0.33 \\
\hline $\operatorname{Mix} \operatorname{GED}(2,2)$ & 0.07 & 0.18 & 0.11 & 0.08 & 0.30 & 0.22 & 0.20 \\
\hline $\operatorname{Mix} \operatorname{GED}(3,2)$ & 0.08 & 0.15 & 0.12 & 0.09 & 0.34 & 0.10 & 0.34 \\
\hline $\operatorname{Mix} \operatorname{GED}(3,3)$ & 0.07 & 0.11 & 0.14 & 0.13 & 0.32 & 0.20 & 0.33 \\
\hline $\operatorname{Mix} \operatorname{GED}(4,4)$ & 0.07 & 0.12 & 0.08 & 0.11 & 0.29 & 0.13 & 0.36 \\
\hline $\mathrm{A}^{1} \operatorname{MixStable}(2,2)$ & 0.04 & 0.17 & 0.20 & 0.12 & 0.33 & 0.10 & 0.21 \\
\hline $\mathrm{A}^{1} \operatorname{MixStable}(3,2)$ & 0.05 & 0.16 & 0.14 & 0.09 & 0.34 & 0.13 & 0.26 \\
\hline $\mathrm{A}^{1} \operatorname{MixStable}(3,3)$ & 0.06 & 0.16 & 0.14 & 0.10 & 0.30 & 0.15 & 0.25 \\
\hline $\mathrm{A}^{1} \operatorname{MixStable}(4,4)$ & 0.07 & 0.07 & 0.10 & 0.07 & 0.28 & 0.21 & 0.32 \\
\hline $\mathrm{A}^{2} \operatorname{MixStable}(2,2)$ & 0.18 & 0.09 & 0.09 & 0.07 & 0.11 & 0.07 & 0.26 \\
\hline $\mathrm{A}^{2} \operatorname{MixStable}(3,2)$ & 0.19 & 0.08 & 0.10 & 0.08 & 0.10 & 0.05 & 0.27 \\
\hline $\mathrm{A}^{2} \operatorname{MixStable}(3,3)$ & 0.19 & 0.11 & 0.10 & 0.07 & 0.11 & 0.05 & 0.28 \\
\hline $\mathrm{A}^{2} \operatorname{MixStable}(4,4)$ & 0.19 & 0.08 & 0.09 & 0.07 & 0.11 & 0.05 & 0.27 \\
\hline \multicolumn{8}{|l|}{$5 \% \mathrm{VaR}$} \\
\hline $\operatorname{MixNormal}(2,2)$ & 0.19 & 0.36 & 0.22 & 0.31 & 0.34 & 0.53 & 0.20 \\
\hline $\operatorname{MixNormal}(3,2)$ & 0.17 & 0.25 & 0.16 & 0.28 & 0.47 & 0.69 & 0.49 \\
\hline $\operatorname{MixNormal}(3,3)$ & 0.10 & 0.23 & 0.10 & 0.28 & 0.47 & 0.62 & 0.53 \\
\hline $\operatorname{MixNormal}(4,4)$ & 0.20 & 0.22 & 0.10 & 0.27 & 0.42 & 0.59 & 0.64 \\
\hline $\operatorname{Mix} \operatorname{GED}(2,2)$ & 0.20 & 0.27 & 0.16 & 0.31 & 0.40 & 0.31 & 0.35 \\
\hline $\operatorname{Mix} \operatorname{GED}(3,2)$ & 0.19 & 0.29 & 0.12 & 0.26 & 0.46 & 0.54 & 0.60 \\
\hline $\operatorname{Mix} \operatorname{GED}(3,3)$ & 0.16 & 0.23 & 0.12 & 0.28 & 0.47 & 0.33 & 0.61 \\
\hline $\operatorname{Mix} \operatorname{GED}(4,4)$ & 0.17 & 0.22 & 0.10 & 0.27 & 0.44 & 0.37 & 0.64 \\
\hline $\mathrm{A}^{1} \operatorname{MixStable}(2,2)$ & 0.25 & 0.26 & 0.14 & 0.31 & 0.42 & 0.54 & 0.28 \\
\hline $\mathrm{A}^{1} \operatorname{MixStable}(3,2)$ & 0.16 & 0.24 & 0.13 & 0.29 & 0.45 & 0.66 & 0.40 \\
\hline $\mathrm{A}^{1} \operatorname{MixStable}(3,3)$ & 0.16 & 0.18 & 0.10 & 0.30 & 0.49 & 0.60 & 0.56 \\
\hline $\mathrm{A}^{1} \operatorname{MixStable}(4,4)$ & 0.17 & 0.16 & 0.09 & 0.28 & 0.42 & 0.63 & 0.65 \\
\hline $\mathrm{A}^{2} \operatorname{MixStable}(2,2)$ & 0.30 & 0.22 & 0.17 & 0.25 & 0.55 & 0.34 & 0.31 \\
\hline $\mathrm{A}^{2} \operatorname{MixStable}(3,2)$ & 0.30 & 0.19 & 0.18 & 0.26 & 0.58 & 0.41 & 0.35 \\
\hline $\mathrm{A}^{2} \operatorname{MixStable}(3,3)$ & 0.31 & 0.18 & 0.21 & 0.24 & 0.57 & 0.42 & 0.33 \\
\hline $\mathrm{A}^{2} \operatorname{MixStable}(4,4)$ & 0.31 & 0.19 & 0.20 & 0.24 & 0.59 & 0.41 & 0.31 \\
\hline
\end{tabular}

Table 6: Integrated root mean squared error of the VaR prediction up to the $1 \%$ and $5 \%$ level for all models under study (with $\delta=1$ ). Entries in boldface denote the best estimate. Results are based on the same out-of-sample exercise as in Table 3 . 


\begin{tabular}{|c|c|c|c|c|c|c|c|}
\hline model & DAX & $\mathrm{S} \& \mathrm{P}$ & DJIA & NIKKEI & $¥ / €$ & $\$ / €$ & NASDAQ \\
\hline \multicolumn{8}{|c|}{ Unconditional Coverage, $\mathrm{LR}_{\mathrm{UC}}$} \\
\hline $\operatorname{MixNormal}(2,2)$ & $3.1 \mathrm{e}-4$ & 0.14 & 0.88 & 0.68 & $3.23^{*}$ & 0.28 & 0.05 \\
\hline $\operatorname{MixNormal}(3,2)$ & 0.05 & 0.14 & 0.03 & 0.68 & $4.14^{* *}$ & 0.07 & $5.62^{* *}$ \\
\hline $\operatorname{MixNormal}(3,3)$ & 1.07 & 0.14 & 0.32 & 1.07 & $4.14^{* *}$ & 0.28 & $6.81^{* * *}$ \\
\hline $\operatorname{MixNormal}(4,4)$ & 0.39 & 0.32 & 0.03 & 0.39 & $4.14^{* *}$ & 0.28 & $5.62 * *$ \\
\hline $\operatorname{Mix} \operatorname{GED}(2,2)$ & $3.1 \mathrm{e}-4$ & 0.14 & 0.32 & 0.39 & $5.16^{* *}$ & 1.70 & 1.07 \\
\hline $\operatorname{Mix} \operatorname{GED}(3,2)$ & 0.05 & 0.56 & 0.14 & 0.68 & $4.14^{* *}$ & 0.07 & $4.57^{* *}$ \\
\hline $\operatorname{Mix} \operatorname{GED}(3,3)$ & 0.68 & 0.14 & $3.1 \mathrm{e}-4$ & 1.56 & $5.16^{* *}$ & 1.11 & $5.62 * *$ \\
\hline $\operatorname{Mix} \operatorname{GED}(4,4)$ & 0.68 & 0.14 & $3.1 \mathrm{e}-4$ & 0.68 & $5.16^{* *}$ & 0.28 & $4.57^{* *}$ \\
\hline $\mathrm{A}^{1} \operatorname{MixStable}(2,2)$ & 0.32 & 0.32 & 0.14 & 0.68 & $5.16^{* *}$ & 0.28 & $3.65^{*}$ \\
\hline $\mathrm{A}^{1} \operatorname{MixStable}(3,2)$ & 0.14 & 0.56 & $3.1 e-4$ & 0.68 & $3.23^{*}$ & 0.07 & $4.57^{* *}$ \\
\hline $\mathrm{A}^{1} \operatorname{MixStable}(3,3)$ & $3.1 e-4$ & 0.32 & 0.56 & 0.68 & $4.14^{* *}$ & 0.28 & $2.84^{*}$ \\
\hline $\mathrm{A}^{1} \operatorname{MixStable}(4,4)$ & 0.68 & 0.03 & $3.1 \mathrm{e}-4$ & 0.39 & $4.14^{* *}$ & 0.28 & $3.65^{*}$ \\
\hline $\mathrm{A}^{2} \operatorname{MixStable}(2,2)$ & 1.56 & 0.05 & 0.03 & 0.39 & 1.70 & 0.07 & $2.84^{*}$ \\
\hline $\mathrm{A}^{2} \operatorname{MixStable}(3,2)$ & 1.56 & 0.05 & 0.14 & 0.39 & 1.70 & 0.07 & $3.65^{*}$ \\
\hline $\mathrm{A}^{2}$ MixStable $(3,3)$ & 1.56 & 0.05 & 0.03 & 0.05 & 1.70 & 0.07 & $4.57^{* *}$ \\
\hline $\mathrm{A}^{2}$ MixStable $(4,4)$ & 1.56 & 0.05 & 0.03 & 0.17 & 1.70 & 0.07 & $3.65^{*}$ \\
\hline \multicolumn{8}{|c|}{ Independence, $\mathrm{LR}_{\text {IND }}$} \\
\hline MixNormal $(2,2)$ & 1.28 & 0.63 & 0.77 & 0.39 & 1.06 & 0.37 & 0.50 \\
\hline $\operatorname{MixNormal}(3,2)$ & 1.40 & 0.63 & 0.59 & 0.39 & 0.92 & 0.33 & 0.19 \\
\hline $\operatorname{MixNormal}(3,3)$ & 1.98 & 0.63 & 0.67 & 0.36 & 0.92 & 0.37 & 0.16 \\
\hline $\operatorname{MixNormal}(4,4)$ & 1.67 & 0.67 & 0.59 & 0.43 & 0.92 & 0.37 & 0.19 \\
\hline $\operatorname{Mix} \operatorname{GED}(2,2)$ & 1.28 & 0.63 & 0.67 & 0.43 & 0.79 & 0.53 & 0.36 \\
\hline $\operatorname{Mix} \operatorname{GED}(3,2)$ & 1.40 & 0.72 & 0.63 & 0.39 & 0.92 & 0.33 & 0.21 \\
\hline $\operatorname{Mix} \operatorname{GED}(3,3)$ & 1.82 & 0.63 & 0.54 & 0.32 & 0.79 & 0.48 & 0.19 \\
\hline $\operatorname{Mix} \operatorname{GED}(4,4)$ & 1.82 & 0.63 & 0.54 & 0.39 & 0.79 & 0.37 & 0.21 \\
\hline $\mathrm{A}^{1} \operatorname{MixStable}(2,2)$ & 0.96 & 0.67 & 0.63 & 0.39 & 0.79 & 0.37 & 0.24 \\
\hline $\mathrm{A}^{1} \operatorname{MixStable}(3,2)$ & 1.06 & 0.72 & 0.54 & 0.39 & 1.06 & 0.33 & 0.21 \\
\hline $\mathrm{A}^{1} \operatorname{MixStable}(3,3)$ & 1.28 & 0.67 & 0.72 & 0.39 & 0.92 & 0.37 & 0.26 \\
\hline $\mathrm{A}^{1} \operatorname{MixStable}(4,4)$ & 1.82 & 0.59 & 0.54 & 0.43 & 0.92 & 0.37 & 0.24 \\
\hline $\mathrm{A}^{2} \operatorname{MixStable}(2,2)$ & 2.15 & 0.50 & 0.59 & 0.43 & 1.37 & 0.33 & 0.26 \\
\hline $\mathrm{A}^{2}$ MixStable $(3,2)$ & 2.15 & 0.50 & 0.63 & 0.43 & 0.53 & 0.33 & 0.24 \\
\hline $\mathrm{A}^{2} \operatorname{MixStable}(3,3)$ & 2.15 & 0.50 & 0.59 & 0.50 & 1.37 & 0.33 & 0.21 \\
\hline $\mathrm{A}^{2} \operatorname{MixStable}(4,4)$ & 2.15 & 0.50 & 0.59 & 0.46 & 1.37 & 0.33 & 0.24 \\
\hline \multicolumn{8}{|c|}{ Conditional Coverage, $\mathrm{LR}_{\mathrm{CC}}$} \\
\hline $\operatorname{MixNormal}(2,2)$ & 1.28 & 0.77 & 1.65 & 1.08 & 4.28 & 0.66 & 0.55 \\
\hline $\operatorname{MixNormal}(3,2)$ & 1.45 & 0.77 & 0.62 & 1.08 & 5.06 & 0.40 & 5.81 \\
\hline $\operatorname{MixNormal}(3,3)$ & 3.05 & 0.77 & 0.99 & 1.43 & 5.06 & 0.66 & $6.97^{*}$ \\
\hline $\operatorname{MixNormal}(4,4)$ & 2.06 & 0.99 & 0.62 & 0.81 & 5.06 & 0.66 & 5.81 \\
\hline $\operatorname{Mix} \operatorname{GED}(2,2)$ & 1.28 & 0.77 & 0.99 & 0.81 & 5.95 & 2.24 & 1.43 \\
\hline $\operatorname{Mix} \operatorname{GED}(3,2)$ & 1.45 & 1.29 & 0.77 & 1.08 & 5.06 & 0.40 & 4.78 \\
\hline $\operatorname{Mix} \operatorname{GED}(3,3)$ & 2.50 & 0.77 & 0.54 & 1.89 & 5.95 & 1.58 & 5.81 \\
\hline $\operatorname{Mix} \operatorname{GED}(4,4)$ & 2.50 & 0.77 & 0.54 & 1.08 & 5.95 & 0.66 & 4.78 \\
\hline $\mathrm{A}^{1} \operatorname{MixStable}(2,2)$ & 1.28 & 0.99 & 0.77 & 1.08 & 5.95 & 0.66 & 3.88 \\
\hline $\mathrm{A}^{1} \operatorname{MixStable}(3,2)$ & 1.20 & 1.29 & 0.54 & 1.08 & 4.28 & 0.40 & 4.78 \\
\hline $\mathrm{A}^{1}$ MixStable $(3,3)$ & 1.28 & 0.99 & 1.29 & 1.08 & 5.06 & 0.66 & 3.11 \\
\hline $\mathrm{A}^{1} \operatorname{MixStable}(4,4)$ & 2.50 & 0.62 & 0.54 & 0.81 & 5.06 & 0.66 & 3.88 \\
\hline $\mathrm{A}^{2} \operatorname{MixStable}(2,2)$ & 3.71 & 0.55 & 0.62 & 0.81 & 3.08 & 0.40 & 3.11 \\
\hline $\mathrm{A}^{2} \operatorname{MixStable}(3,2)$ & 3.71 & 0.55 & 0.77 & 0.81 & 2.24 & 0.40 & 3.88 \\
\hline $\mathrm{A}^{2} \operatorname{MixStable}(3,3)$ & 3.71 & 0.55 & 0.62 & 0.55 & 3.08 & 0.40 & 4.78 \\
\hline $\mathrm{A}^{2} \operatorname{MixStable}(4,4)$ & 3.71 & 0.55 & 0.62 & 0.64 & 3.08 & 0.40 & 3.88 \\
\hline
\end{tabular}

Table 7: Test statistics at the $1 \%$-VaR level, $\mathrm{LR}_{\mathrm{CC}}=\mathrm{LR}_{\mathrm{UC}}+\mathrm{LR}_{\mathrm{IND}}$, as described in Christoffersen (1998) for all models under study (with $\delta=1$ ). Entries in boldface denote the best outcomes. ***, $* *$, and $*$ denote significance at the $1 \%, 5 \%$, and $10 \%$ levels, respectively. Results are based on the same out-of-sample exercise as in Table 3. 